\title{
EQUIDISTRIBUTION AND GENERALIZED MAHLER MEASURES
}

\author{
L. SZPIRO AND T. J. TUCKER
}

\begin{abstract}
If $K$ is a number field and $\varphi: \mathbb{P}_{K}^{1} \longrightarrow \mathbb{P}_{K}^{1}$ is a rational map of degree $d>1$, then at each place $v$ of $K$, one can associate to $\varphi$ a generalized Mahler measure for polynomials $F \in K[t]$. These Mahler measures give rise to a formula for the canonical height $h_{\varphi}(\beta)$ of an element $\beta \in \bar{K}$; this formula generalizes Mahler's formula for the usual Weil height $h(\beta)$. In this paper, we use diophantine approximation to show that the generalized Mahler measure of a polynomial $F$ at a place $v$ can be computed by averaging $\log |F|_{v}$ over the periodic points of $\varphi$.
\end{abstract}

This paper is dedicated to the memory of Serge Lang, who taught the world number theory for more than fifty years, through his research, lectures, and books.

The usual Weil height of a rational number $x / y$, where $x$ and $y$ are integers without a common prime factor, is defined as

$$
h(x / y)=\log \max (|x|,|y|) .
$$

More generally, one can define the usual Weil height $h(\beta)$ of an algebraic number $\beta$ in a number field $K$ by summing $\log \max \left(|\beta|_{v},|1|\right)$ over all of the absolute values $v$ of $K$. Mahler ([Mah60]) has proven that if $F$ is a nonzero irreducible polynomial in $\mathbb{Z}[t]$ with coprime coefficients such that $F(\beta)=0$, then

$$
\operatorname{deg}(F) h(\beta)=\int_{0}^{1} \log \left|F\left(e^{2 \pi i \theta}\right)\right| d \theta .
$$

The quantity $\int_{0}^{1} \log \left|F\left(e^{2 \pi i \theta}\right)\right| d \theta$ is often referred to as the Mahler measure of $F$.

It is easy to see that $h\left(\beta^{2}\right)=2 h(\beta)$ for any algebraic number $\beta$. Similarly, it is easy to check that for any continuous function $g$ on the unit circle, we have

$$
\int_{0}^{1} g\left(\left(e^{2 \pi i \theta}\right)^{2}\right) d \theta=\int_{0}^{1} g\left(e^{2 \pi i \theta}\right) d \theta
$$

2000 Mathematics Subject Classification. Primary 11G50, Secondary 11J68, 37F10.

Key words and phrases. Height functions, Mahler measure, dynamical systems, periodic points, equidistribution, diophantine approximation.

The first author was partially supported by NSF Grant 0071921. The second author was partially supported by NSF Grant 0101636. 
Furthermore, the unit circle is the Julia set of $\varphi$. Thus, Mahler's formula says that one obtains the height of an algebraic number by integrating its minimal polynomial against the unique measure $\mu$ such that $\varphi^{*} \mu=\mu$ and $\mu$ is supported on the Julia set of $\varphi$.

Now, let $\varphi: \mathbb{P}_{\mathbb{C}}^{1} \longrightarrow \mathbb{P}_{\mathbb{C}}^{1}$ be any nonconstant rational map. Brolin ([Bro65]) and Lyubich ([Lyu83]) have constructed a totally $\varphi$-invariant probability measure $\mu_{\varphi}$ (that is, we have $\varphi^{*} \mu$ and $\varphi_{*} \mu$ ) with support on the Julia set of $\varphi$; Freire, Lopes, and Mañe ([FLM83]) have demonstrated that this measure is the unique totally $\varphi$-invariant probability measure with support on the Julia set of $\varphi$. When $\varphi$ is defined over a number field $K$, Call and Silverman ([CS93]) have constructed a height function $h_{\varphi}$ with the properties that: (1) $h_{\varphi}(\varphi(x))=(\operatorname{deg} \varphi) h_{\varphi}(x)$ and $(2)$ there is a constant $C_{\varphi}$ such that $\mid h(x)-$ $h_{\varphi}(x) \mid<C_{\varphi}$ for all $x \in \mathbb{P}^{1}(\bar{K})$. In [PST04], it is shown that Mahler's formula (0.0.1) generalizes to the adelic formula

$$
(\operatorname{deg} F) h_{\varphi}(x)=\sum_{\text {places } v \text { of } K} \int_{\mathbb{P}^{1}\left(\mathbb{C}_{v}\right)} \log |F|_{v} d \mu_{\varphi, v},
$$

where $\beta$ is an algebraic point, $F$ is a nonzero irreducible polynomial in $\mathbb{Q}[t]$ such that $F(\beta)=0$, the measure $\mu_{\varphi, v}$ at an archimedean place is the totally $\varphi$-invariant probability measure constructed by Brolin and Lyubich, and the integral $\int_{\mathbb{P}^{1}\left(\mathbb{C}_{v}\right)} \log |F|_{v} d \mu_{\varphi, v}$ at a finite place $v$ is defined so that its value is the $v$-adic analog of the value at an archimedean place (note that as defined in [PST04], these are not integrals per se). Favre and Rivera-Letelier have also given a proof of 0.0.2, using actual integrals on Berkovich spaces; Piñeiro ([Piñ05]) and Chambert-Loir and Thuillier ([CLT04, Thu06]) have recently proven higher-dimensional generalizations of 0.0.2.

Lyubich [Lyu83] has also proven that for any continuous function $g$ and any archimedean place $v$, the integrals $\int_{\mathbb{P}^{1}\left(\mathbb{C}_{v}\right)} g d \mu_{\varphi, v}$ can be computed by averaging $g$ on the periodic points of $\varphi$; that is to say,

$$
\lim _{k \rightarrow \infty} \frac{1}{(\operatorname{deg} \varphi)^{k}} \sum_{\varphi^{k}(w)=w} g(w)=\int_{\mathbb{P}^{1}\left(\mathbb{C}_{v}\right)} g d \mu_{\varphi, v} .
$$

Autissier ([Aut01]), Bilu ([Bi197]), Szpiro, Ullmo, and Zhang ([SUZ97]), and others have obtained generalizations and variations of this result. The most recent generalization, proven independently by Baker and Rumely ([BR06]), Chambert-Loir ([CL06]), and Favre and Rivera-Letelier ([FRL04] and [FRL07]) states that (0.0.3) continues to hold when the periodic points $w$ such that $\varphi^{k}(w)=w$ are replaced by the conjugates of any infinite nonrepeating sequence of algebraic points with height tending to 0 and when the measure $\mu_{\varphi, v}$ is the unique totally $\varphi$-invariant measure without point masses on the $v$-adic Berkovich space (see [Ber90]) for a finite place $v$.

The function $\log |F|$, for $F$ a nonconstant polynomial, is not continuous in general, of course. Thus, the equidistribution results cited above do not allow us to compute Mahler measures by averaging $\log |F|_{v}$ over points of 
small height. One can, however, show that for any $\beta \in \overline{\mathbb{Q}}$, we have

$$
[\mathbb{Q}(\beta): \mathbb{Q}] h(\beta)=\lim _{k \rightarrow \infty} \frac{1}{d^{k}} \sum_{\xi^{n}=1} \log |F(\xi)|=\int_{0}^{1} \log \left|F\left(e^{2 \pi i \theta}\right)\right| d \theta,
$$

where $F$ is a nonzero irreducible polynomial in $\mathbb{Z}[t]$ with coprime coefficients such that $F(\beta)=0$ (see [EW99, Chapter 1], [Sch74]). Everest, Ward, and Ní Fhlathúin have proved similar results for maps that come from multiplication on an elliptic curve ([EW99, Chapter 6], [EF96]). The proofs of these results make use of the theory of linear forms in logarithms ([Bak75], [Dav95]), which is used to show that the periodic points of the maps in question have strong diophantine properties. It is not clear how to apply the theory of linear forms in logarithms in the case of more general rational maps. In this paper, we use Roth's Theorem ([Rot55]) from diophantine approximation in place of the theory of linear forms in logarithms. This allows us to work in greater generality.

0.1. Statements of the main theorems. The main results of this paper extend (0.0.4) to a formula that holds for all rational maps. Let $K$ be a number field or a function field of characteristic zero, let $v$ be a place of $K$, and let $\varphi: \mathbb{P}_{K}^{1} \longrightarrow \mathbb{P}_{K}^{1}$ be a nonconstant rational map of degree $d>1$. We prove the following equidistribution result for the periodic points of $\varphi$.

Theorem 4.7. For any nonzero polynomial $F$ with coefficients in $\bar{K}$, we have

$$
\int_{\mathbb{P}^{1}\left(\mathbb{C}_{v}\right)} \log |F|_{v} d \mu_{\varphi, v}=\lim _{k \rightarrow \infty} \frac{1}{d^{k}} \sum_{\substack{\varphi^{k}([w: 1])=[w: 1] \\ F(w) \neq 0}} \log |F(w)|_{v} .
$$

This allows us to show that for any point $\beta \in \bar{K}$, the canonical height $h_{\varphi}(\beta)$ can be computed by taking the average of the log of the absolute value of a minimal polynomial for $\beta$ over the periodic points of $\varphi$.

Theorem 4.10. For any $\beta \in \bar{K}$ and any nonzero irreducible $F \in K[t]$ such that $F(\beta)=0$, we have

$$
\begin{aligned}
(\operatorname{deg} K) & (\operatorname{deg} F)\left(h_{\varphi}(\beta)-h_{\varphi}(\infty)\right) \\
& =\sum_{\text {places } v \text { of } K} \lim _{k \rightarrow \infty} \frac{1}{d^{k}} \sum_{\begin{array}{c}
\varphi^{k}([w: 1])=[w: 1] \\
F(w) \neq 0
\end{array}} \log |F(w)|_{v} .
\end{aligned}
$$

In both the theorems, the $w$ are counted with multiplicity. We explain what multiplicity means in this context in Section 1.

We are also able to prove that $\int_{\mathbb{P}^{1}\left(\mathbb{C}_{v}\right)} \log |F|_{v} d \mu_{\varphi, v}$ is the limit as $n$ goes to infinity of the average of $\log |F|_{v}$ on the points $w$ for which $\varphi^{n}(w)=\alpha$, where $\alpha$ is an algebraic point that is not an exceptional point for $\varphi$. We state this in Theorem 4.6. This enables us to prove Theorem 4.9, which is the analog of Theorem 4.10 for the points $w$ such that $\varphi^{n}(w)=\alpha$. 
0.2. Outline of the paper. This paper is organized as follows:

1 - Notation and terminology.

2 - Brolin-Lyubich integrals and local heights.

3 - Preliminaries from diophantine approximation.

4 - Main results: 4.1 - Using Roth's Theorem; 4.2 - Preperiodic points; 4.3 - Proofs of the main theorems.

5 - A counterexample.

6 - Applications: 6.1 - Lyapunov exponents; 6.2 - Symmetry of canonical heights; 6.3 - Computing with points of small height.

The strategy of the proof of the main theorems is fairly simple. By additivity, it suffices to prove our results for polynomials of the form $F(t)=t-\beta$ for $\beta \in \bar{K}$. After Section 2, we are reduced to showing that

$$
\begin{aligned}
\lim _{k \rightarrow \infty} \frac{1}{d^{k}} \sum_{\substack{\varphi^{k}([w: 1])=[w: 1] \\
w \neq \beta}} \log |w-\beta|_{v} & =\lim _{k \rightarrow \infty} \frac{\log \max \left(\left|P_{k}(\beta, 1)\right|_{v},\left|Q_{k}(\beta, 1)\right|_{v}\right)}{d^{k}} \\
& -\lim _{k \rightarrow \infty} \frac{\log \max \left(\left|P_{k}(1,0)\right|_{v},\left|Q_{k}(1,0)\right|_{v}\right)}{d^{k}},
\end{aligned}
$$

where $\varphi^{k}$ is written as

$$
\varphi^{k}\left(\left[T_{0}: T_{1}\right]\right)=\left[P_{k}\left(T_{0}, T_{1}\right): Q_{k}\left(T_{0}, T_{1}\right)\right]
$$

for coprime homogeneous polynomials $P_{k}$ and $Q_{k}$ in the $K\left[T_{0}, T_{1}\right]$. The points $w$ for which $\varphi^{k}(w)=w$ are just the solutions to the equation $P_{k}(w, 1)$ $w Q_{k}(w, 1)=0$. Thus, we get the left-hand side of $(0.0 .5)$ by taking the limit of $\log \left|P_{k}(\beta, 1)-\beta Q_{k}(\beta, 1)\right|_{v} / d^{k}$ as $k$ goes to $\infty$. For each $k$, we rewrite this as

$$
\frac{\log \left|Q_{k}(\beta, 1)\right|_{v}}{d^{k}}+\frac{\log \left|\frac{P_{k}(\beta, 1)}{Q_{k}(\beta, 1)}-\beta\right|_{v}}{d^{k}}
$$

and use diophantine approximation to show that the second term in the equation above usually goes to 0 as $k \rightarrow \infty$; our theorems then follow after a bit of calculation. The diophantine approximation result we use is Roth's Theorem, which we state in Section 3 as Theorem 3.1. We use Roth's Theorem to derive Lemma 4.2, which is the key lemma in our proofs of the main theorems. The idea for the proof of Lemma 4.2 comes from Siegel's famous paper [Sie29]. We should note that after writing this paper we discovered that Silverman ([Sil93]) has used methods very similar to those found here at the beginning of Section 4; we require a slight modification of his results along these lines, however, so we present the necessary argument here in full.

Propositions 4.4 and 4.5 deal with the additional complications that may arise when the $\beta$ in (0.0.5) is preperiodic. These complications are overcome with somewhat lengthy - but essentially basic - calculations that are very 
similar to some of the computations carried out by Morton and Silverman in [MS95].

In Section 5, we construct a simple counterexample that shows that Theorem 4.7 will not hold in general when the polynomial $F$ does not have algebraic coefficients (it is likely that the theorem will also fail if the point $\alpha$ is not algebraic). We construct a transcendental number $\beta$ such that the $\operatorname{limit}_{\lim _{k \rightarrow \infty} \frac{1}{2^{k}} \sum_{\xi^{2}}=1} \log |\xi-\beta|$ does not exist. This means that there is no way to prove the main results of this paper without using some special properties of algebraic numbers.

Acknowledgments. We would like to thank M. Baker, A. Chambert-Loir, L. DeMarco, R. Rumely, and S. Zhang for many helpful conversations. In particular, we thank M. Baker, L. DeMarco, and R. Rumely for suggesting some of the applications mentioned in Section 6. 6.

\section{Notation AND TERMinOLOGY}

We fix the following notation:

- $K$ is a number field or a function field of characteristic 0 (by function field we mean a finite algebraic extension of a field of the form $K_{\text {cons }}(T)$ where $K_{\text {cons }}$ is algebraically closed in $K$ );

- $v$ is a place of $K$;

- $K_{v}$ is the completion of $K$ at $v$;

- $\mathbb{C}_{v}$ is the completion of an algebraic closure of $K_{v}$ at $v$;

- $\bar{K}$ is the algebraic closure of $K$ in $\mathbb{C}_{v}$ (note that this means that $v$ extends to all of $\bar{K})$;

- $n_{v}=\left[K_{v}: \mathbb{Q}_{v}\right]$ if $K$ is a number field;

- $n_{v}=1$ if $K$ is a function field;

- $\operatorname{deg} K=[K: \mathbb{Q}]$ if $K$ is a number field;

- $\operatorname{deg} K=1$ if $K$ is a function field.

We let $|\cdot|_{v}$ be an absolute value on $\mathbb{C}_{v}$ corresponding to $v$. When $K$ is a function field and $\pi_{v}$ generates the maximal prime $\mathcal{M}_{v}$ in the local ring $\mathfrak{o}_{v}$ corresponding to $v$, we specify that

$$
\left|\pi_{v}\right|_{v}=e^{-\left[\left(\mathfrak{o}_{v} / \mathcal{M}_{v}\right): K_{\mathrm{cons}}\right]},
$$

where $K_{\text {cons }}$ is the field of constants in $K$. When $K$ is a number field and $v$ is nonarchimedean, we normalize $|\cdot|_{v}$ so that

$$
|p|_{v}=p^{-n_{v}}
$$

when $v$ lies over $p$. When $K$ is a number field and $v$ is archimedean we normalize so that $|\cdot|_{v}=|\cdot|^{n_{v}}$ on $\mathbb{Q}$, where $|\cdot|$ is the usual archimedean absolute value on $\mathbb{Q}$.

Throughout this paper, we will work with a nonconstant morphism $\varphi$ : $\mathbb{P}_{K}^{1} \longrightarrow \mathbb{P}_{K}^{1}$ of degree $d>1$. We choose homogeneous polynomials $P, Q \in$ 
$K\left[T_{0}, T_{1}\right]$ of degree $d$ without a common factor along with a coordinate system $[s: t]$ for $\mathbb{P}_{\bar{K}}^{1}$ such that

$$
\varphi\left(\left[T_{0}: T_{1}\right]\right)=\left[P\left(T_{0}, T_{1}\right): Q\left(T_{0}, T_{1}\right)\right],
$$

where $P$ and $Q$ have no common zero in $\mathbb{P}^{1}(\bar{K})$. We let $P_{1}=P$ and $Q_{1}=Q$, and for $k \geq 2$ we define $P_{k}$ and $Q_{k}$ recursively by

$$
P_{k}\left(T_{0}, T_{1}\right)=P_{k-1}\left(P\left(T_{0}, T_{1}\right), Q\left(T_{0}, T_{1}\right)\right)
$$

and

$$
Q_{k}\left(T_{0}, T_{1}\right)=Q_{k-1}\left(P\left(T_{0}, T_{1}\right), Q\left(T_{0}, T_{1}\right)\right) .
$$

Having chosen coordinates, we can define the usual Weil height as

$$
h([a: b])=\frac{1}{\operatorname{deg} K} \sum_{\text {places } v \text { of } K} \log \max \left(|a|_{v},|b|_{v}\right)
$$

when $a, b \in K$. When $a$ and $b$ lie in an extension $L$ of $K$, this definition extends to

$$
h([a: b])=\frac{1}{[L: K](\operatorname{deg} K)} \sum_{\text {places } w \text { of } L}\left[L_{w}: K_{v}\right] \log \max \left(|a|_{w},|b|_{w}\right),
$$

where $L_{w}$ is the completion of $L$ at $w$ and the absolute value $\mid \cdot{ }_{w}$ restricts to some $|\cdot|_{v}$ on $K$.

As in [CS93], we define the canonical height $h_{\varphi}$ as

$$
h_{\varphi}([a: b])=\lim _{k \rightarrow \infty} \frac{h\left(\varphi^{k}([a: b])\right)}{d^{k}} .
$$

We say that $\alpha \in \mathbb{P}^{1}(\bar{K})$ is a periodic point for $\varphi$ if there exists a positive integer $n$ such that $\varphi^{n}(\alpha)=\alpha$. If $\alpha$ is periodic, we define the period of $\alpha$ to be the smallest positive integer $\ell$ such that $\varphi^{\ell}(\alpha)=\alpha$. We say that $\alpha$ is preperiodic if there exists a positive integer $n$ such that $\varphi^{n}(\alpha)$ is periodic.

We will use a small amount of the theory of dynamics on the projective plane; for a more thorough account of the subject, we refer the reader to Milnor's ([Mil99]) and Beardon's ([Bea91]) books on the subject. We say that $\alpha \in \mathbb{P}^{1}(\bar{K})$ is an exceptional point for $\varphi$ if $\varphi^{2}(\alpha)=\alpha$ and $\varphi^{2}$ is totally ramified at $\alpha$. This is equivalent to saying that the set $\bigcup_{k=1}^{\infty}\left(\varphi^{k}\right)^{-1}(\alpha)$ is finite (see [Bea91, Chapter 4.1]). If $\alpha$ is exceptional, then at each place $v$, there is a maximal $v$-adically open set $\mathcal{U}$ containing $\alpha$ such that the sequence $\left(\varphi^{\ell k}(\beta)\right)_{k}$ converges to $\alpha$ for each $\beta \in \mathcal{U}$, where $\ell$ is the period of $\alpha$ (which is either 1 or 2). We call $\mathcal{U}$ the attracting basin of $\alpha$ (see [Bea91, Chapter 6.3], which uses the terminology "local basin").

We always count points with multiplicities in this paper. The multiplicity of a point $[z: 1]$ in the multi-set $\left\{w \mid \varphi^{k}(w)=w\right\}$ is the highest power of $t-z$ that divides the polynomial $P_{k}(t, 1)-t Q_{k}(t, 1)$. The multiplicity of a point $[z: 1]$ in the multi-set $\left\{w \mid \varphi^{k}(w)=[s: u]\right\}$ is the highest power of $t-z$ that divides the polynomial $u P_{k}(t, 1)-s Q_{k}(t, 1)$ (here $s, u$, and $z$ are taken to be elements of $\bar{K}$, while $t$ is taken to be a variable). 
We note that everything done in this paper depends upon our choice of coordinates. In particular, our integrals are closely related to the canonical local heights (see [CG97]) for the point [1:0] at infinitely, so our choice of the point at infinity affects all of our integrals. To emphasize the fact that we treat $[1: 0]$ as the point at infinity, we denote it as $\infty$ where appropriate.

\section{Brolin-Lyubich INTEGRAls AND LOCAL HEIGHTS}

We will work with the limits

$$
\lim _{k \rightarrow \infty} \frac{\log \max \left(\left|P_{k}(a, b)\right|_{v},\left|Q_{k}(a, b)\right|_{v}\right)}{d^{k}}
$$

for $(a, b) \in \mathbb{C}_{v} \backslash\{(0,0)\}$. For a proof that these limits exist, see [PST04], [BR06], or [CG97] (the proof is essentially an exercise in using telescoping sums and geometric series). Note that Call and Goldstine ([CG97, Theorem 3.1]) have shown that

$$
\hat{h}_{\varphi, v}([\beta: 1])=\lim _{k \rightarrow \infty} \frac{\log \max \left(\left|P_{k}(\beta, 1)\right|_{v},\left|Q_{k}(\beta, 1)\right|_{v}\right)}{d^{k}}
$$

is the unique Weil function for [1:0] at $v$ (see [Lan83, Chapter 10] for a definition of Weil functions) that satisfies

$$
\hat{h}_{\varphi, v}(\varphi([a: b]))=d \hat{h}_{\varphi, v}([a: b])+\log \left|Q\left(\frac{a}{b}, 1\right)\right|_{v},
$$

for any $[a: b] \neq[1: 0]$ (see [CG97, Theorem 2.1]). The function $\hat{h}_{\varphi, v}(\cdot)$ is called a canonical local height for $\varphi$. We also note that these local heights can also be constructed by taking a quantity obtained from the "FubiniStudy" metric and passing to the limit; specifically, the limit in (2.0.8) is also equal to

$$
\lim _{k \rightarrow \infty} \frac{\log \sqrt{\left.\left|P_{k}(a, b)\right|_{v}^{2}+\left|Q_{k}(a, b)\right|_{v}\right)^{2}}}{d^{k}} .
$$

The equality follows from the uniqueness of the Call-Goldstine local height or from the arguments in [Zha95, Section 2]. Note that Baker and Rumely ([BR06]) use (2.0.9) to form local heights.

As noted in the introduction, Brolin [Bro65] and Lyubich [Lyu83] have constructed a totally $\varphi$-invariant measure $\mu_{\varphi, v}$ with support on the Julia set of $\varphi$, when $v$ is an infinite place (see also [FLM83]). More recently, Baker and Rumely ([BR06]), Chambert-Loir ([CL06]), and Favre and Rivera-Letelier ([FRL04] and [FRL07]) have constructed a $\varphi$-invariant measure $\mu_{\varphi, v}$ on the Berkovich space associated to $\mathbb{P}^{1}\left(\mathbb{C}_{v}\right)$; this measure is unique among $\varphi$ invariant measures without point masses the Berkovich space associated to $\mathbb{P}^{1}\left(\mathbb{C}_{v}\right)$. 
Proposition 2.1. Let $v$ be a place of a number field $K$ and let $F(t)=t-\beta$ for $\beta \in \mathbb{C}_{v}$. Then

$$
\begin{aligned}
\int_{\mathbb{P}^{1}\left(\mathbb{C}_{v}\right)} \log |F|_{v} d \mu_{\varphi, v} & =\lim _{k \rightarrow \infty} \frac{\log \max \left(\left|P_{k}(\beta, 1)\right|_{v},\left|Q_{k}(\beta, 1)\right|_{v}\right)}{d^{k}} \\
& -\lim _{k \rightarrow \infty} \frac{\log \max \left(\left|P_{k}(1,0)\right|_{v},\left|Q_{k}(1,0)\right|_{v}\right)}{d^{k}} .
\end{aligned}
$$

Proof. We will prove this following the methods of Baker and Rumely, who show that the measures $\mu_{\varphi, v}$ are Laplacians of local height functions. The proposition could also be proved using the work of Favre and Rivera-Letelier ([FRL07]) or Chambert-Loir and Thuillier ([CLT04, Thu06]), who proved more general Mahler formulas (but do not formulate them in terms of limits such as (2.0.8)). In [BR06], Baker and Rumely show that for $w \in \mathbb{C}_{v}$, the function $H_{w}$ defined by

$$
H_{w}([a: b])=-\log (w b-a)+\lim _{k \rightarrow \infty} \frac{\log \sqrt{\left.\left|P_{k}(a, b)\right|_{v}^{2}+\left|Q_{k}(a, b)\right|_{v}\right)^{2}}}{d^{k}}
$$

is subharmonic on $\mathbb{P}^{1}\left(\mathbb{C}_{v}\right) \backslash\{[w: 1]\}$ (see [BR06, BR04]). Furthermore, they show that if $\Delta$ is the distributional Laplacian (i.e. $-d d^{c}$ considered in the distributional sense, which can be extended to the setting of Berkovich spaces as described in [BR04]), then

$$
\frac{1}{n_{v} p(v)} \Delta H_{w}=-\mu_{\varphi, v}+\delta_{w}
$$

where $\delta_{w}$ is the usual Dirac point mass at $w$ and $p(v)$ is the log of the characteristic of the residue field of $v$ when $v$ nonarchimedean, and is simply 1 when $v$ is archimedean. Similarly, we have

$$
\frac{1}{n_{v} p(v)} \Delta \log |t-\beta|_{v}=\delta_{[1: 0]}-\delta_{\beta}
$$

(see [FRL07, Section 5.1] or the same reasoning that gives (2.1.2)). Now, since $\log |t-\beta|_{v}$ and $H_{w}$ are both subharmonic on $\mathbb{P}^{1}\left(\mathbb{C}_{v}\right) \backslash\{[1: 0],[w$ : $1],[\beta: 1]\}$ we have

$$
\begin{aligned}
\int_{\mathbb{P}^{1}\left(\mathbb{C}_{v}\right)} \log |t-\beta|_{v} d \mu_{\varphi, v} & =\left(\int_{\mathbb{P}^{1}\left(\mathbb{C}_{v}\right)} \log |t-\beta|_{v}\left(-\frac{1}{n_{v} p(v)} \Delta H_{w}\right)\right)+\log |w-\beta|_{v} \\
& =\left(\int_{\mathbb{P}^{1}\left(\mathbb{C}_{v}\right)} H_{w}\left(-\frac{1}{n_{v} p(v)} \Delta \log |t-\beta|_{v}\right)\right)+\log |w-\beta|_{v} \\
& =H_{w}([\beta: 1])-H_{w}([1: 0])+\log |w-\beta|_{v}
\end{aligned}
$$


Since (2.0.8) and (2.0.9) are equal by the discussion above, (4.6.3) becomes

$$
\begin{aligned}
-\log |w-\beta|_{v}+ & \lim _{k \rightarrow \infty} \frac{\log \max \left(\left|P_{k}(\beta, 1)\right|_{v},\left|Q_{k}(\beta, 1)\right|_{v}\right)}{d^{k}}+\log |1| \\
& -\lim _{k \rightarrow \infty} \frac{\log \max \left(\left|P_{k}(1,0)\right|_{v},\left|Q_{k}(1,0)\right|_{v}\right)}{d^{k}}+\log |w-\beta|_{v} \\
=\lim _{k \rightarrow \infty} \frac{\log \max \left(\left|P_{k}(\beta, 1)\right|_{v},\left|Q_{k}(\beta, 1)\right|_{v}\right)}{d^{k}} & -\lim _{k \rightarrow \infty} \frac{\log \max \left(\left|P_{k}(1,0)\right|_{v},\left|Q_{k}(1,0)\right|_{v}\right)}{d^{k}}
\end{aligned}
$$

as desired.

Note that although our integrals are defined for points in $\mathbb{C}_{v}$, the results we prove in Section 4 only apply to points in $\bar{K}$. Note as well that we make no use of the fact that our limits correspond to actual integrals, either in the proofs of our main theorems or in the applications in Section 6 .

When $K$ is a function field, it should also be possible to construct suitable integrals at the places of $K$. Since this has not yet been done, however, we will have to make do with a definition rather than a proof.

Definition 2.2. Let $v$ be a place of a function field $K$ and let $F(t)=t-\beta$ for $\beta \in \mathbb{C}_{v}$. Then

$$
\begin{aligned}
\int_{\mathbb{P}^{1}\left(\mathbb{C}_{v}\right)} \log |F|_{v} d \mu_{\varphi, v} & =\lim _{k \rightarrow \infty} \frac{\log \max \left(\left|P_{k}(\beta, 1)\right|_{v},\left|Q_{k}(\beta, 1)\right|_{v}\right)}{d^{k}} \\
& -\lim _{k \rightarrow \infty} \frac{\log \max \left(\left|P_{k}(1,0)\right|_{v},\left|Q_{k}(1,0)\right|_{v}\right)}{d^{k}} .
\end{aligned}
$$

\section{Preliminaries from diophantine approximation}

The following well-known theorem of Roth $([\operatorname{Rot} 55])$ is the principal tool from diophantine approximation that is used in this paper.

Theorem 3.1. (Roth). If $\alpha \in \mathbb{C}$ is algebraic over $\mathbb{Q}$, then for any $\epsilon>0$, there is a constant $C$ such that

$$
\left|\alpha-\frac{a}{b}\right|>\frac{C}{|b|^{2+\epsilon}}
$$

for all $a / b \in \mathbb{Q}$ such that $a / b \neq \alpha$.

We will need to work in slightly greater generality. In the terminology of the previous section, Roth's admits the following generalization (see [Lan83, Theorem 7.1.1]), which holds when $K$ is number field or a function fields of characteristic 0 .

Theorem 3.2. Let $\alpha_{1}, \ldots, \alpha_{n}$ be elements of $\bar{K}$ and let $L \subset \bar{K}$ be a finite extension of $K$. Then, for any $\epsilon>0$ and any places $v$ of $K$ and $w$ of $L$ such 
that $w \mid v$, we have

$$
\frac{1}{[L: K](\operatorname{deg} K)} \sum_{i=1}^{n} \max \left(0,-\log \left|\alpha_{i}-\beta\right|_{v}^{\left[L_{w}: K_{v}\right] n_{v}}\right) \leq(2+\epsilon) h(\beta)+O(1),
$$

for all $\beta \in L$ not in the set $\left\{\alpha_{1}, \ldots, \alpha_{n}\right\}$.

Let $[a: 1]$ be a point in $\mathbb{P}^{1}(\bar{K})$. Then for any $[b: 1] \neq[a: 1]$ in $\mathbb{P}^{1}\left(\mathbb{C}_{v}\right)$, we let

$$
\lambda_{[a: 1], v}([b: 1])=\max \left(-\log |b-a|_{v}, 0\right) .
$$

We extend this definition to the point at $[1: 0]$ by letting

$$
\lambda_{[a: 1], v}([1: 0])=0 .
$$

and

$$
\lambda_{[1: 0], v}([b: 1])=\max \left(0, \log |b|_{v}\right) .
$$

We will work with divisors on $\mathbb{P} \frac{1}{K}$ rather than elements of $\bar{K}$. Let $D=$ $\sum_{i=1}^{n} m_{i} \alpha_{i}$, where $\alpha_{i} \in \mathbb{P}^{1}(\bar{K})$ and $m_{i} \in \mathbb{Z}$. We let

$$
\lambda_{D, v}(\beta)=\sum m_{i} \lambda_{\alpha_{i}, v}(\beta)
$$

for points $\beta \in \mathbb{P}^{1}\left(\mathbb{C}_{v}\right)$ that are not in Supp $D$. Then $\lambda_{D, v}$ is a Weil function for $D$ at $v$ as defined in [Lan83, Chapter 10]. It is easy to check that for any divisor $D$ and any rational map $\varphi$ on $\mathbb{P}^{1}$, we have

$$
\lambda_{D, v}(\varphi(\beta))=\lambda_{\varphi^{*} D, v}(\beta)+O(1),
$$

for all $\beta \in \mathbb{P}^{1}(\bar{K})$ away from the support of $D$ and $\varphi^{*} D$. This is a general functorial property of Weil functions, as explained in [Lan83, Chapter 10].

For a divisor $D=\sum_{i=1}^{n} m_{i} \alpha_{i}$, where $\alpha_{i} \in \mathbb{P}^{1}(\bar{K})$, we define

$$
r(D)=\max _{i}\left(m_{i}\right) .
$$

With this terminology, it follows from Theorem 3.2 that for any $\epsilon>0$, any finite extension $L$ of $K$, and any positive divisor $D$ on $\mathbb{P}^{1}(\bar{K})$ with $r(D)=1$, we have

$$
\frac{1}{[L: K](\operatorname{deg} K)} \lambda_{D, v}(\beta) \leq(2+\epsilon) h(\beta)+O(1)
$$

for all $\beta \in \mathbb{P}^{1}(L)$ away from the support of $D$. Hence, for any positive divisor $D$ we have

$$
\frac{1}{[L: K](\operatorname{deg} K)} \lambda_{D, v}(\beta) \leq r(D)(2+\epsilon) h(\beta)+O(1) .
$$




\section{MAIN RESUlts}

We begin with a simple Lemma on how $r\left(\left(\varphi^{n}\right)^{*}(D)\right.$ behaves as $n \rightarrow \infty$ when $D$ is a divisor that does not contain an exceptional point of $\varphi$. We recall that in general if $D=\sum_{i=1}^{n} m_{i} \alpha_{i}$ is a divisor on $\mathbb{P}^{1}$ and $\psi: \mathbb{P}^{1} \longrightarrow \mathbb{P}^{1}$ is a nonconstant rational map, then

$$
\psi^{*} D=\sum_{i=1}^{n} \sum_{\psi\left(\beta_{i}\right)=\alpha_{i}} m_{i} e\left(\beta_{i} / \alpha_{i}\right) \beta_{i}
$$

where $e\left(\beta_{i} / \alpha_{i}\right)$ is the ramification index of $\psi$ at $\beta_{i}$.

Lemma 4.1. Let $D$ be a divisor such that $\operatorname{Supp} D$ does not contain any exceptional points of $\varphi$. Then $\lim _{k \rightarrow \infty} \frac{r\left(\left(\varphi^{k}\right)^{*} D\right)}{d^{k}}=0$.

Proof. Recall that $\alpha$ is an exceptional point if and only if $\varphi^{2}(\alpha)=\alpha$ and $\varphi$ is totally ramified at both $\alpha$ and $\varphi(\alpha)$. Since $\varphi$ has at most two totally ramified points, it follows that if $\alpha$ is not exceptional, then one of $\alpha, \varphi(\alpha)$, and $\varphi^{2}(\alpha)$ is not a totally ramified point of $\varphi$. Since the degree of $\varphi^{3}$ is $d^{3}$, this means that for any divisor $E$ such that $\operatorname{Supp} E$ does not contain an exceptional point, we have $r\left(\left(\varphi^{3}\right)^{*} E\right)<d^{3} r(E)$ (by (4.0.4)), so $r\left(\left(\varphi^{3}\right)^{*} E\right) \leq$ $d^{2}(d-1) r(E)$ Now, since $\operatorname{Supp} D$ does not contain an exceptional point, $\operatorname{Supp}\left(\varphi^{k}\right)^{*} D$ does not contain an exceptional point for any $k$. Thus, for any $k \geq 3$, we see that $\frac{r\left(\left(\varphi^{k}\right)^{*} D\right)}{d^{k}}$ is less than or equal to $((d-1) / d)^{(k-2) / 3} r(D)$, which goes to zero as $k$ goes to infinity.

4.1. Using Roth's Theorem. Roth's Theorem allows us to prove the following lemma. The idea of the proof is that if $\varphi^{k+\ell}(\beta)$ approximates $D$ very closely, then $\varphi^{k}(\beta)$ approximates $\left(\varphi^{\ell}\right)^{*} D$ very closely. Since $\varphi^{k}(\beta)$ has height approximately equal to $1 / d^{\ell}$ times the height of $\varphi^{k+\ell}(\beta)$, this makes $h\left(\varphi^{k}(\beta)\right)$ small relative to $\lambda_{\left(\varphi^{\ell}\right)^{*} D}(\beta)$. Repeating this for infinitely many $\varphi^{k}(\beta)$ gives a contradiction to Roth's Theorem. This idea is due to Siegel ([Sie29]); similar arguments can be found in [Sil93].

Lemma 4.2. Let $D$ be a positive divisor on $\mathbb{P}^{1}$ such that $\operatorname{Supp} D$ does not contain any of the exceptional points of $\varphi$. Let $\beta$ be a point in $\mathbb{P}^{1}(\bar{K})$ for which there is a strictly increasing sequence of integers $\left(e_{i}\right)_{i=1}^{\infty}$ such that $\varphi^{e_{i}}(\beta) \notin \operatorname{Supp} D$. Then

$$
\lim _{i \rightarrow \infty} \frac{\lambda_{D, v}\left(\varphi^{e_{i}}(\beta)\right)}{d^{e_{i}}}=0 .
$$

Proof. Let $L$ be a finite extension of $K$ for which $\beta \in \mathbb{P}^{1}(L)$. Choose $\delta>0$. By Lemma 4.1, we may pick an integer $\ell$ such that $\frac{r\left(\left(\varphi^{\ell}\right)^{*} D\right)}{d^{\ell}}<\delta / 2$. We may then write $\frac{r\left(\left(\varphi^{\ell}\right)^{*} D\right)(2+\epsilon)}{d^{\ell}}=\delta$ for some $\epsilon>0$. For any $e_{i}$, we have $\varphi^{e_{i}-\ell}(\beta) \notin \operatorname{Supp}\left(\varphi^{\ell}\right)^{*} D$ since $\varphi^{e_{i}}(\beta) \notin \operatorname{Supp} D$. Thus, applying Roth's 
Theorem (as expressed in (3.2.3)), we find that for all $e_{i}$ we have

$$
\frac{1}{[L: K](\operatorname{deg} K)} \lambda_{\left(\varphi^{\ell}\right)^{*} D, v}\left(\varphi^{e_{i}-\ell}(\beta)\right) \leq r\left(\left(\varphi^{\ell}\right)^{*} D\right)(2+\epsilon) h\left(\varphi^{e_{i}-\ell}(\beta)\right)+O(1) .
$$

Using (3.2.2) and the fact that $h\left(\varphi^{e_{i}}(\beta)\right) \leq d^{\ell} h_{\varphi}\left(\varphi^{e_{i}-\ell}(\beta)\right)+O(1)$, we then obtain

$$
\begin{aligned}
\frac{1}{[L: K](\operatorname{deg} K)} \lambda_{D, v}\left(\varphi^{e_{i}}(\beta)\right) & \leq \frac{1}{[L: K](\operatorname{deg} K)} \lambda_{\left(\varphi^{\ell}\right)^{*} D, v}\left(\varphi^{e_{i}-\ell}(\beta)\right)+O(1) \\
& \leq r\left(\left(\varphi^{\ell}\right)^{*} D\right)(2+\epsilon) h\left(\varphi^{e_{i}-\ell}(\beta)\right)+O(1) \\
& \leq \frac{r\left(\left(\varphi^{\ell}\right)^{*} D\right)(2+\epsilon)}{d^{\ell}} h\left(\varphi^{e_{i}}(\beta)\right)+O(1) \\
& \leq \delta h\left(\varphi^{e_{i}}(\beta)\right)+O(1) \\
& \leq \delta d^{e_{i}} h(\beta)+O(1) .
\end{aligned}
$$

Dividing through by $d^{e_{i}}$ gives

$$
\lim _{i \rightarrow \infty} \sup \frac{\lambda_{D, v}\left(\varphi^{e_{i}}(\beta)\right)}{d^{e_{i}}} \leq[L: K](\operatorname{deg} K) \delta h(\beta) .
$$

Since $\lambda_{D, v}\left(\varphi^{e_{i}}(\beta)\right) \geq 0$, letting $\delta$ go to zero gives (4.2.1), as desired.

This allows us to prove the following Proposition, which will be used to prove Theorems 4.6 and 4.7 .

Proposition 4.3. Let $\alpha=[s: u]$ be a nonexceptional point in $\mathbb{P}^{1}(\bar{K})$. Then for any point $\beta=[a: b]$ in $\mathbb{P}^{1}(\bar{K})$ and any strictly increasing sequence of integers $\left(e_{i}\right)_{i=1}^{\infty}$ such that $\varphi^{e_{i}}(\beta) \neq \alpha$, we have

$$
\lim _{i \rightarrow \infty} \frac{\log \left|u P_{e_{i}}(a, b)-s Q_{e_{i}}(a, b)\right|_{v}}{d^{e_{i}}}=\lim _{i \rightarrow \infty} \frac{\log \max \left(\left|P_{e_{i}}(a, b)\right|_{v},\left|Q_{e_{i}}(a, b)\right|_{v}\right)}{d^{e_{i}}} .
$$

Proof. Note that we know that the the limit on the right-hand side of the equation above exists by the discussion at the beginning of Section 2 .

If $[1: 0]$ is an exceptional point of $\varphi$, let $\mathcal{U}$ be its attracting basin; if $[1: 0]$ is not exceptional, let $\mathcal{U}$ simply equal $\{[1: 0]\}$. We will divide $\left(e_{i}\right)_{i=1}^{\infty}$ into two subsequences: one consisting of the $e_{i}$ for which $\varphi^{e_{i}}(\beta) \notin \mathcal{U}$ and one consisting of the remaining integers in the sequence $\left(e_{i}\right)_{i=1}^{\infty}$. Let $\left(\ell_{j}\right)_{j=1}^{\infty}$ be the subsequence consisting of all integers $\ell_{j}$ in $\left(e_{i}\right)_{i=1}^{\infty}$ such that $\varphi^{\ell_{j}}(\beta) \notin \mathcal{U}$ (this subsequence may be empty). We have

$$
\lim _{j \rightarrow \infty} \frac{\max \left(\log \left|P_{\ell_{j}}(a, b) / Q_{\ell_{j}}(a, b)\right|_{v}, 0\right)}{d^{\ell_{j}}}=0 .
$$

If $[1: 0]$ is not exceptional, this follows from Lemma 4.2 applied to $D=[1$ : $0]$, along with (3.2.1). If [1:0] is exceptional, the fact that $\varphi^{\ell_{j}}(\beta) \notin \mathcal{U}$ for all $j$ implies that $\left|P_{\ell_{j}}(a, b) / Q_{\ell_{j}}(a, b)\right|_{v}$ is bounded for all $j$, so (4.3.1) clearly holds. It follows immediately from (4.3.1) that

$$
\lim _{j \rightarrow \infty} \frac{\log \max \left(\left|P_{\ell_{j}}(a, b)\right|_{v},\left|Q_{\ell_{j}}(a, b)\right|_{v}\right)}{d^{\ell_{j}}}=\lim _{j \rightarrow \infty} \frac{\log \left|Q_{\ell_{j}}(a, b)\right|_{v}}{d^{\ell_{j}}} .
$$


Note that if $u=0$, then

$$
u P_{\ell_{j}}(a, b)-s Q_{\ell_{j}}(a, b)=s Q_{\ell_{j}}(a, b),
$$

so we are done. Otherwise, by Lemma 4.2, we have

$$
\lim _{j \rightarrow \infty} \frac{\max \left(0,-\log \left|\frac{P_{\ell_{j}}(a, b)}{Q_{\ell_{j}}(a, b)}-\frac{s}{u}\right|_{v}\right)}{d^{\ell_{j}}}=0 .
$$

Combining this with (4.3.1), we see that

$$
\lim _{j \rightarrow \infty} \frac{\log \left|\frac{P_{\ell_{j}}(a, b)}{Q_{\ell_{j}}(a, b)}-\frac{s}{u}\right|_{v}}{d^{\ell_{j}}}=0 .
$$

Thus, using (4.3.2), we obtain

$$
\begin{aligned}
\lim _{j \rightarrow \infty} & \frac{\log \left|u P_{\ell_{j}}(a, b)-s Q_{\ell_{j}}(a, b)\right|_{v}}{d^{\ell_{j}}} \\
& =\lim _{j \rightarrow \infty} \frac{\log \left(\left|Q_{\ell_{j}}(a, b)\right|_{v}|u|_{v}\left|\frac{P_{\ell_{j}}(a, b)}{Q_{\ell_{j}}(a, b)}-\frac{s}{u}\right|_{v}\right)}{d^{\ell_{j}}} \\
& =\lim _{j \rightarrow \infty} \frac{\log \left|Q_{\ell_{j}}(a, b)\right|_{v}}{d^{\ell_{j}}}+\lim _{j \rightarrow \infty} \frac{\log \left|\frac{P_{\ell_{j}}(a, b)}{Q_{\ell_{j}}(a, b)}-\frac{s}{u}\right|_{v}}{d^{\ell_{j}}} \\
& =\lim _{j \rightarrow \infty} \frac{\log \max \left(\left|P_{\ell_{j}}(a, b)\right|_{v},\left|Q_{\ell_{j}}(a, b)\right|_{v}\right)}{d^{\ell_{j}}},
\end{aligned}
$$

as desired.

Now, let $\left(m_{j}\right)_{j=1}^{\infty}$ be be the subsequence of $\left(e_{i}\right)_{i=1}^{\infty}$ consisting of all integers $m_{j}$ in $\left(e_{i}\right)_{i=1}^{\infty}$ such that $\varphi^{m_{j}}(\beta) \in \mathcal{U}$ (this subsequence may also be empty). If $\alpha=[1: 0]$, then $[1: 0]$ is not exceptional by assumption, so there are no $m_{j}$ and we are done. Otherwise, we have

$$
\lim _{j \rightarrow \infty} \frac{\left|s Q_{m_{j}}(a, b)\right|_{v}}{\left|u P_{m_{j}}(a, b)\right|_{v}}=0
$$

since $\frac{P_{m_{j}}(a, b)}{Q_{m_{j}}(a, b)}$ goes to infinity and $u \neq 0$. This implies that

$$
\begin{aligned}
& \lim _{j \rightarrow \infty} \frac{\log \left|u P_{m_{j}}(a, b)-s Q_{m_{j}}(a, b)\right|_{v}}{d^{m_{j}}} \\
& =\lim _{j \rightarrow \infty} \frac{\log \left|u P_{m_{j}}(a, b)\right|_{v}}{d^{m_{j}}} \\
& =\lim _{j \rightarrow \infty} \frac{\left.\log \max \left(\left|P_{m_{j}}(a, b)\right|_{v},\left|Q_{m_{j}}(a, b)\right|_{v}\right)\right)}{d^{m_{j}}} .
\end{aligned}
$$

Since every element of the sequence $\left(e_{i}\right)_{i=1}^{\infty}$ is in $\left(\ell_{j}\right)_{j=1}^{\infty}$ or $\left(m_{j}\right)_{j=1}^{\infty}$, this completes our proof. 
4.2. Preperiodic points. Proposition 4.3 provides all the information we need when $\varphi^{k}([a: b])=[s: u]$ for at most finitely many $k$; this will always be the case when $[s: u]$ is not preperiodic. When $[s: u]$ is preperiodic, however, there may be infinitely many $k$ such that $\varphi^{k}([a: b])=[s: u]$. New complications arise when this is the case; we treat these complications in Propositions 4.4 and 4.5 .

Suppose that $\left(b T_{0}-a T_{1}\right)^{w_{k}}$ is the highest power of $\left(b T_{0}-a T_{1}\right)$ that divides $u P_{k}\left(T_{0}, T_{1}\right)-s Q_{k}\left(T_{0}, T_{1}\right)$ in $\bar{K}\left[T_{0}, T_{1}\right]$. We write

$$
u P_{k}\left(T_{0}, T_{1}\right)-s Q_{k}\left(T_{0}, T_{1}\right)=\left(b T_{0}-a T_{1}\right)^{w_{k}} G_{k}\left(T_{0}, T_{1}\right)
$$

where $G_{k}$ is a polynomial in $\bar{K}\left[T_{0}, T_{1}\right]$ such that $G_{k}(a, b) \neq 0$.

Proposition 4.4. Let $[s: u]$ be a nonexceptional point of $\varphi$. Then, with notation as above, we have

$$
\lim _{k \rightarrow \infty} \frac{\log \left|G_{k}(a, b)\right|_{v}}{d^{k}}=\lim _{k \rightarrow \infty} \frac{\log \max \left(\left|P_{k}(a, b)\right|_{v},\left|Q_{k}(a, b)\right|_{v}\right)}{d^{k}} .
$$

Proof. By Proposition 4.3, equation (4.4.1) holds if we restrict to the $k$ for which $\varphi^{k}([a: b]) \neq \alpha$. If there are only finitely many $k$ such that $\varphi^{k}([a: b])=\alpha$, we are therefore done. Otherwise, let $j$ be the smallest positive integer such that $\varphi^{j}([\beta: 1])=\alpha$ and let $\ell$ be the period of $\alpha$. Then $\varphi^{k}([\beta: 1])=\alpha$ precisely when $k$ is of the form $j+m \ell$ for some integer $m \geq 0$. If $\varphi^{\ell}([s: u])=[s: u]$, then $u T_{0}-s T_{1}$ divides $u P_{\ell}\left(T_{0}, T_{1}\right)-s Q_{\ell}\left(T_{0}, T_{1}\right)$.

Suppose that $u \neq 0$. Then, expanding $Q_{\ell}$ out in the variables $u T_{0}-s T_{1}$ and $T_{1}$, we see that since $u T_{0}-s T_{1}$ cannot divide $Q_{\ell}\left(T_{0}, T_{1}\right)$ (because if it did, then it would also divide $P_{\ell}\left(T_{0}, T_{1}\right)$ and we know that $Q_{\ell}$ and $P_{\ell}$ have no factors), we have

$$
Q_{\ell}\left(T_{0}, T_{1}\right)=g_{0} T_{1}^{d^{\ell}}+\left(u T_{0}-s T_{1}\right) W\left(T_{0}, T_{1}\right)
$$

for some nonzero $g_{0} \in \bar{K}$ and some $W\left(T_{0}, T_{1}\right) \in \bar{K}\left[T_{0}, T_{1}\right]$. For any $m \geq 1$ we thus have

$$
Q_{m \ell}=g_{0}\left(Q_{(m-1) \ell}\right)^{d^{\ell}}+\left(u P_{(m-1) \ell}-s Q_{(m-1) \ell}\right) W\left(P_{(m-1) \ell}, Q_{(m-1) \ell}\right) .
$$

Using induction, we see then that

$$
Q_{m \ell}\left(T_{0}, T_{1}\right)=g_{0}^{\sum_{i=0}^{m-1} d^{i \ell}} T_{1}^{d^{m \ell}}+\left(u T_{0}-s T_{1}\right) W_{m}\left(T_{0}, T_{1}\right),
$$

for some polynomial $W_{m}\left(T_{0}, T_{1}\right) \in \bar{K}\left[T_{0}, T_{1}\right]$. Similarly, we may write

$$
\begin{aligned}
& u P_{\ell}\left(T_{0}, T_{1}\right)-s Q_{\ell}\left(T_{0}, T_{1}\right) \\
& =\left(u T_{0}-s T_{1}\right)^{r} f_{r} T_{1}^{d-r}+\left(u T_{0}-s T_{1}\right)^{r+1} V\left(T_{0}, T_{1}\right),
\end{aligned}
$$

for some nonzero $f_{r} \in \bar{K}$, some integer $r>0$, and some $V\left(T_{0}, T_{1}\right)$ in $\bar{K}\left[T_{0}, T_{1}\right]$. Since $[s: u]$ is not an exceptional point of $\varphi$, we have $r<d^{\ell}$ (note that if $r$ were to equal to $d^{\ell}$, then $\varphi$ would have to ramify totally at $\varphi([s: u]), \ldots, \varphi^{\ell}[s: u]$, which would imply that $\ell=2$ and that $[s: u]$ is 
therefore an exceptional point, as explained in Section 1). Then for any $m$, we have

$$
\begin{aligned}
u P_{m \ell}-s Q_{m \ell} & =\left(u P_{(m-1) \ell}-s Q_{(m-1) \ell}\right)^{r} f_{r} Q_{(m-1) \ell}^{d-r} \\
& +\left(P_{(m-1) \ell}-s Q_{(m-1) \ell}\right)^{r+1} V\left(P_{(m-1) \ell}, Q_{(m-1) \ell}\right),
\end{aligned}
$$

so, using (4.4.2), (4.4.3), and induction, we obtain

$$
\begin{aligned}
& u P_{m \ell}\left(T_{0}, T_{1}\right)-s Q_{m \ell}\left(T_{0}, T_{1}\right) \\
& =\left(u T_{0}-s T_{1}\right)^{r^{m}} f_{r}^{\sum_{i=0}^{m-1} r^{i}} T_{1}^{d^{m \ell}-r^{m}} g_{0}^{\sum_{i=0}^{m-1}\left(d^{i \ell}-r^{i}\right)} \\
& +\left(u T_{0}-s T_{1}\right)^{r^{m}+1} Z_{m}\left(T_{0}, T_{1}\right),
\end{aligned}
$$

for $Z_{m}$ a polynomial in $\bar{K}\left[T_{0}, T_{1}\right]$. Since $r<d^{\ell}$, we have

$$
\lim _{m \rightarrow \infty} \frac{\log \left|f_{r}^{\sum_{i=0}^{m-1} r^{i}} g_{0}^{\sum_{i=0}^{m-1}\left(d^{i \ell}-r^{i}\right)}\right|_{v}}{d^{m \ell}}=\lim _{m \rightarrow \infty} \frac{\log \left|g_{0}^{\sum_{i=0}^{m-1} d^{i \ell}}\right|_{v}}{d^{m \ell}}=\frac{\log \left|g_{0}\right|_{v}}{d^{\ell}-1} .
$$

Now, let $\epsilon$ be the highest power of $a T_{0}-b T_{1}$ that divides $u P_{j}-s Q_{j}$. Using (4.4.4), we see that we have

$$
u P_{j+m \ell}\left(T_{0}, T_{1}\right)-s Q_{j+m \ell}\left(T_{0}, T_{1}\right)=\left(b T_{0}-a T_{1}\right)^{\epsilon r^{m}} G_{j+m \ell}\left(T_{0}, T_{1}\right)
$$

for a polynomial $G_{j+m \ell} \in \bar{K}\left[T_{0}, T_{1}\right]$. Letting $m$ go to infinity, we see from (4.4.4) that

$$
\lim _{m \rightarrow \infty} \frac{\log \left|G_{j+m \ell}(a, b)\right|_{v}}{d^{j+m \ell}}=\frac{\log \left|g_{0}\right|_{v}}{d^{j}\left(d^{\ell}-1\right)}+\frac{\log \left|Q_{j}(a, b)\right|_{v}}{d^{j}} .
$$

Similarly, (4.4.2) yields

$$
\lim _{m \rightarrow \infty} \frac{\log \left|Q_{j+m \ell}(a, b)\right|_{v}}{d^{j+m \ell}}=\frac{\log \left|g_{0}\right|_{v}}{d^{j}\left(d^{\ell}-1\right)}+\frac{\log \left|Q_{j}(a, b)\right|_{v}}{d^{j}} .
$$

Moreover, since $u P_{j+m \ell}(a, b)=s Q_{j+m \ell}(a, b)$ for every $m$, we have

Hence

$$
\lim _{m \rightarrow \infty} \frac{\log \left|P_{j+m \ell}(a, b)\right|_{v}}{d^{j+m \ell}}=\lim _{m \rightarrow \infty} \frac{\log \left|Q_{j+m \ell}(a, b)\right|_{v}}{d^{j+m \ell}} .
$$

$$
\lim _{m \rightarrow \infty} \frac{\log \left|G_{j+m \ell}(a, b)\right|_{v}}{d^{j+m \ell}}=\lim _{m \rightarrow \infty} \frac{\log \max \left(\left|P_{j+m \ell}(a, b)\right|_{v},\left|Q_{j+m \ell}(a, b)\right|_{v}\right)}{d^{j+m \ell}},
$$

which completes our proof in the case $u \neq 0$. The proof in the case $u=0$ proceeds in exactly the same way, using $T_{0}$ in place of $T_{1}$.

We have a similar result for the polynomials $T_{0} P_{k}-T_{1} Q_{k}$. We write

$$
T_{0} P_{k}\left(T_{0}, T_{1}\right)-T_{1} Q_{k}\left(T_{0}, T_{1}\right)=\left(b T_{0}-a T_{1}\right)^{n_{k}} H_{k}\left(T_{0}, T_{1}\right)
$$

where $H_{k}$ is a polynomial in $\bar{K}\left[T_{0}, T_{1}\right]$ such that $H_{k}(a, b) \neq 0$. The proof of the following proposition is similar to Morton's and Silverman's proof of [MS95, Lemma 3.4], but it requires a bit more detail since it yields information about $H_{k}(a, b)$ as well as $n_{k}$. 
Proposition 4.5. With notation as above, we have

$$
\lim _{k \rightarrow \infty} \frac{\log \left|H_{k}(a, b)\right|_{v}}{d^{k}}=\lim _{k \rightarrow \infty} \frac{\log \max \left(\left|P_{k}(a, b)\right|_{v},\left|Q_{k}(a, b)\right|_{v}\right)}{d^{k}} .
$$

Furthermore, $n_{k}$ remains bounded as $k$ goes to infinity.

Proof. If $\left(e_{i}\right)_{i=1}^{\infty}$ is a strictly increasing sequence of integers such that $\varphi^{e_{i}}([a$ : $b]) \neq[a: b]$ for each $e_{i}$, then

$$
H_{e_{i}}\left(T_{0}, T_{1}\right)=T_{0} P_{e_{i}}\left(T_{0}, T_{1}\right)-T_{1} Q_{e_{i}}\left(T_{0}, T_{1}\right)
$$

for all $e_{i}$. Hence, by Proposition 4.3, we

$$
\lim _{i \rightarrow \infty} \frac{\log \left|H_{e_{i}}(a, b)\right|_{v}}{d^{e_{i}}}=\lim _{i \rightarrow \infty} \frac{\log \max \left(\left|P_{e_{i}}(a, b)\right|_{v},\left|Q_{e_{i}}(a, b)\right|_{v}\right)}{d^{e_{i}}} .
$$

If $[a: b]$ is not periodic, this finishes the proof. Thus, we may assume that $[a: b]$ is periodic. The rest of the proof is a computation. We divide it into three steps.

Step I. We begin by changing variables so that $[a: b]$ becomes $[0: 1]$. If $b=0$, we write $U_{0}=T_{1} / a$ and $U_{1}=-T_{0}$. We then let

$$
R\left(U_{0}, U_{1}\right)=\frac{1}{a} Q\left(T_{0}, T_{1}\right)
$$

and

$$
S\left(U_{0}, U_{1}\right)=-P\left(T_{0}, T_{1}\right)
$$

(this is simply the inverse of the transformation we defined on $T_{0}$ and $T_{1}$ - our change of variables is obtained by conjugation by a change-of-basis matrix). If $b \neq 0$, we write $U_{1}=\frac{1}{b} T_{1}$ and

$$
U_{0}=b T_{0}-a T_{1}
$$

We then let $S\left(U_{0}, U_{1}\right)=Q\left(T_{0}, T_{1}\right) / b$ and

$$
R\left(U_{0}, U_{1}\right)=b P\left(T_{0}, T_{1}\right)-a Q\left(T_{0}, T_{1}\right) .
$$

We define $R_{k}$ and $S_{k}$ recursively by letting $R_{1}=R, S_{1}=S$, and setting

$$
R_{k+1}\left(U_{0}, U_{1}\right)=R_{k}\left(R\left(U_{0}, U_{1}\right), S\left(U_{0}, U_{1}\right)\right)
$$

and

$$
S_{k+1}\left(U_{0}, U_{1}\right)=S_{k}\left(R\left(U_{0}, U_{1}\right), S\left(U_{0}, U_{1}\right)\right) .
$$

By the construction of our change of variables, we have

$$
U_{1} R_{k}\left(U_{0}, U_{1}\right)-U_{0} S_{k}\left(U_{0}, U_{1}\right)=T_{0} P_{k}\left(T_{0}, T_{1}\right)-T_{1} Q_{k}\left(T_{0}, T_{1}\right)
$$

as polynomials in $T_{0}$ and $T_{1}$. Hence, if $U_{0}^{n_{k}}$ is the highest power of $U_{0}$ that divides $U_{1} R_{k}\left(U_{0}, U_{1}\right)-U_{0} S_{k}\left(U_{0}, U_{1}\right)$ and $\tau_{k}$ is the coefficient of the $U_{0}^{n_{k}} U_{1}^{d^{k}-n_{k}}$ term in $U_{1} R_{k}\left(U_{0}, U_{1}\right)-U_{0} S_{k}\left(U_{0}, U_{1}\right)$, then

$$
\tau_{k}=H_{k}(a, b) .
$$


Now, let $\ell$ be the smallest positive integer for which $\varphi^{\ell}([a: b])=[a: b]$. Note that $\left|S_{m \ell}(1,0)\right|_{v}=\frac{\left|Q_{m \ell}(a, b)\right|_{v}}{|b|_{v}}$ if $b \neq 0$ and $\left|S_{m \ell}(1,0)\right|_{v}=\left|P_{m \ell}(a, b)\right|_{v} /|a|_{v}$ otherwise. Since

$$
\left[P_{m \ell}(a, b): Q_{m \ell}(a, b)\right]=[a: b]
$$

for every $m$, it follows that

$$
\lim _{m \rightarrow \infty} \frac{\log \left|S_{m \ell}(0,1)\right|_{v}}{d^{m \ell}}=\lim _{m \rightarrow \infty} \frac{\log \max \left(\left|P_{m \ell}(a, b)\right|_{v},\left|Q_{m \ell}(a, b)\right|_{v}\right)}{d^{m \ell}} .
$$

Thus, it will suffice to show that

$$
\lim _{m \rightarrow \infty} \frac{\log \left|\tau_{m \ell}\right|_{v}}{d^{m \ell}}=\lim _{m \rightarrow \infty} \frac{\log \left|S_{m \ell}(0,1)\right|_{v}}{d^{m \ell}} .
$$

We write

$$
R_{\ell}\left(U_{0}, U_{1}\right)=\sum_{i=1}^{d^{\ell}} f_{i} U_{0}^{i} U_{1}^{d^{\ell}-i}
$$

(note that $U_{0}$ divides $R_{\ell}$ by our change of variables) and

$$
S_{\ell}\left(U_{0}, U_{1}\right)=\sum_{i=0}^{d^{\ell}} g_{i} U_{0}^{i} U_{1}^{d^{\ell}-i}
$$

Using induction, we see that

$$
R_{m \ell}\left(U_{0}, U_{1}\right) \equiv f_{1}^{m} g_{0}^{\left(\sum_{j=0}^{m-1} d^{j \ell}\right)-m} U_{0} U_{1}^{d^{m \ell}-1} \quad\left(\bmod U_{0}^{2}\right)
$$

and

$$
S_{m \ell}\left(U_{0}, U_{1}\right) \equiv g_{0}^{\sum_{j=0}^{m-1} d^{j \ell}} U_{1}^{d^{m \ell}}\left(\bmod U_{0}^{2}\right)
$$

Thus, we have

$$
\begin{aligned}
& U_{1} R_{m \ell}\left(U_{0}, U_{1}\right)-U_{0} S_{m \ell}\left(U_{0}, U_{1}\right) \\
& \equiv g_{0}^{\sum_{j=0}^{m-1} d^{j \ell}}\left(\left(f_{1} / g_{0}\right)^{m}-1\right) U_{0} U_{1}^{d^{m \ell}}\left(\bmod U_{0}^{2}\right) .
\end{aligned}
$$

Step II. We will now treat the $m$ for which $\left(f_{1} / g_{0}\right)^{m} \neq 1$ We have

$$
|\log |\left(f_{1} / g_{0}\right)^{m}-\left.1\right|_{v} \leq h\left(\left(f_{1} / g_{0}\right)^{m}-1\right) \leq 2 m\left[K\left(f_{1} / g_{0}\right): K\right] h\left(f_{1} / g_{0}\right)
$$

for all $m$ such that $\left(f_{1} / g_{0}\right)^{m} \neq 1$ (this is a simple version of Liouville's theorem), so

$$
\lim _{\substack{m \rightarrow \infty \\\left(f_{1} / g_{0}\right)^{m} \neq 1}} \frac{\log \left|\left(f_{1} / g_{0}\right)^{m}-1\right|_{v}}{d^{m \ell}}=0 .
$$

Thus, dividing (4.5.4) through by $U_{0}$, we obtain

$$
\lim _{\substack{m \rightarrow \infty \\\left(f_{1} / g_{0}\right)^{m} \neq 1}} \frac{\log \left|\tau_{m \ell}\right|_{v}}{d^{m \ell}}=\lim _{m \rightarrow \infty} \frac{\log \left|g_{0}^{\sum_{j=0}^{m-1} d^{j \ell}}\right|_{v}}{d^{m \ell}}=\lim _{m \rightarrow \infty} \frac{\log \left|S_{m \ell}(0,1)\right|_{v}}{d^{m \ell}},
$$

as desired.

Step III. We are left with treating the $m$ for which $\left(f_{1} / g_{0}\right)^{m}=1$. Let $\rho$ be 
the smallest positive integer $m$ such that $\left(f_{1} / g_{0}\right)^{m}=1$ and write $\omega=\rho \ell$. For $q \geq 1$ we write

$$
R_{q \omega}\left(U_{0}, U_{1}\right)=\sum_{i=1}^{d^{q \omega}} x_{i}^{[q]} U_{0}^{i} U_{1}^{d^{q \omega}-i}
$$

(the summation starts at 1 since $U_{0}$ divides $R_{q \omega}$ ) and

$$
S_{q \omega}\left(U_{0}, U_{1}\right)=\sum_{i=0}^{d^{q \omega}} y_{i}^{[q]} U_{0}^{i} U_{1}^{d^{q \omega}-i} .
$$

Since $f_{1}^{\rho}=g_{0}^{\rho}$ by assumption, we have $y_{0}^{[1]}=x_{1}^{[1]}$ by (4.5.4). Multiplying $R_{\omega}$ and $S_{\omega}$ through by a constant will change all of the limits we are calculating by the same fixed amount, so we may assume that $y_{0}^{[1]}=x_{1}^{[1]}=1$. Let $r$ be the smallest integer greater than 0 such that $x_{r}^{[1]} \neq y_{r-1}^{[1]}$ (we have $r \geq 2$ since $\left.\left(f_{1} / g_{0}\right)^{m}=1\right)$. Then $U_{0}^{r}$ divides $U_{1} R_{\omega}-U_{0} S_{\omega}$, which in turn divides $U_{1} R_{q \omega}-U_{0} S_{q \omega}$ for any $q$; hence $U_{0}^{r}$ divides $U_{1} R_{q \omega}-U_{0} S_{q \omega}$ for every $q$, so $x_{j}^{[q]}=y_{j-1}^{[q]}$ for $j<r$. To calculate $x_{r}^{[q]}-y_{r-1}^{[q]}$, we introduce some notation: we let

$$
\left(\sum_{i=0}^{M} t_{i} U_{0}^{i} U_{1}^{M-i}\right)_{j}=t_{j}
$$

for any polynomial $\sum_{i=0}^{M} t_{i} U_{0}^{i} U_{1}^{M-i}$. We have

$$
\begin{aligned}
& x_{r}^{[q]}-y_{r-1}^{[q]} \\
& =\sum_{i=1}^{r} x_{i}^{[q-1]}\left(\left(R_{\omega}\right)^{i}\left(S_{\omega}\right)^{d^{(q-1) \omega}-i}\right)_{r}-\sum_{j=0}^{r-1} y_{j}^{[q-1]}\left(\left(R_{\omega}\right)^{j}\left(S_{\omega}\right)^{d^{(q-1) \omega}-j}\right)_{r-1} .
\end{aligned}
$$

For any $i<r$, we have $x_{i}^{[1]}=y_{i-1}^{[1]}$, so $\left(U_{0} R_{\omega}\right)_{i}=\left(U_{1} S_{\omega}\right)_{i}$. Hence, we have

$$
\left(\left(R_{\omega}\right)^{j}\left(S_{\omega}\right)^{d^{(q-1) \omega}-j}\right)_{r-1}=\left(\left(R_{\omega}\right)^{j+1}\left(S_{\omega}\right)^{d^{(q-1) \omega}-j-1}\right)_{r}
$$

for $j>0$. For $j=0$, we have

$$
\begin{aligned}
\left(S_{\omega}^{d^{(q-1) \omega}}\right)_{r-1} & =\left(\left(R_{\omega}+\left(x_{r}^{[1]}-y_{r-1}^{[1]}\right) U_{0}^{r} U_{1}^{d^{\omega}-r}\right) S_{\omega}^{d^{(q-1) \omega}-1}\right)_{r} \\
& =\left(R_{\omega} S_{\omega}^{d^{(q-1) \omega}-1}\right)_{r}+\left(x_{r}^{[1]}-y_{r-1}^{[1]}\right),
\end{aligned}
$$

since $y_{0}^{[1]}=x_{1}^{[1]}=1$. 
Using equation (4.5.5), we see that

$$
\begin{aligned}
& x_{r}^{[q]}-y_{r-1}^{[q]}=\sum_{i=1}^{r} x_{i}^{[q-1]}\left(\left(R_{\omega}\right)^{i}\left(S_{\omega}\right)^{d^{q \omega}-i}\right)_{r} \\
& -\sum_{j=0}^{r-1} x_{j+1}^{[q-1]}\left(\left(R_{\omega}\right)^{j+1}\left(S_{\omega}\right)^{d^{(q-1) \omega}-j-1}\right)_{r}+\left(x_{r}^{[1]}-y_{r-1}^{[1]}\right)\left(x_{1}^{[q-1]}\right) \\
& +\left(x_{r}^{[q-1]}-y_{r-1}^{[q-1]}\right)\left(\left(R_{\omega}\right)^{r}\left(S_{\omega}\right)^{d^{(q-1) \omega}-r}\right)_{r} \\
& =\left(x_{r}^{[1]}-y_{r-1}^{[1]}\right)\left(x_{1}^{[q-1]}\right)+\left(x_{r}^{[q-1]}-y_{r-1}^{[q-1]}\right),
\end{aligned}
$$

We have $y_{0}^{[q-1]}=x_{1}^{[q-1]}=1$, since $y_{0}^{[1]}=x_{1}^{[1]}=1$. Thus, assuming inductively that

$$
x_{r}^{[q-1]}-y_{r-1}^{[q-1]}=(q-1)\left(x_{r}^{[1]}-y_{r-1}^{[1]}\right),
$$

we have

$$
x_{r}^{[q]}-y_{r-1}^{[q]}=q\left(x_{r}^{[1]}-y_{r-1}^{[1]}\right) .
$$

Note in particular that $n_{q \omega}=r$ for all $q$, so $n_{k}$ is bounded for all $k$, as desired.

Now,

$$
\lim _{q \rightarrow \infty} \frac{\log \left|q\left(x_{r}^{[1]}-y_{r-1}^{[1]}\right)\right|_{v}}{d^{q \omega}}=0
$$

and $\tau_{q \omega}=x_{r}^{[q]}-y_{r-1}^{[q]}$. Since $S_{q \omega}(1,0)$ is simply $y_{0}^{[q-1]}=1$, we have

$$
\lim _{q \rightarrow \infty} \frac{\log \left|\tau_{q \omega}\right|_{v}}{d^{q \omega}}=0=\lim _{q \rightarrow \infty} \frac{\log \left|S_{q \omega}(0,1)\right|_{v}}{d^{q \omega}}
$$

which give us (4.5.3) and thus completes our proof.

4.3. Proofs of the main theorems. Now, we can show that the integral $\int_{\mathbb{P}^{1}\left(\mathbb{C}_{v}\right)} \log |t-\beta|_{v} d \mu_{\varphi, v}$ can be computed by taking the limit of the average of $\log |\beta-w|_{v}$ on the points in $\varphi^{-k}(\alpha)$, as $k \rightarrow \infty$, for any nonexceptional point $\alpha$.

Theorem 4.6. Let $\alpha=[s: u]$ be a nonexceptional point in $\mathbb{P}^{1}(\bar{K})$. Then for any nonzero polynomial $F(t) \in \bar{K}[t]$ we have

$$
\int_{\mathbb{P}^{1}\left(\mathbb{C}_{v}\right)} \log |F|_{v} d \mu_{\varphi, v}=\lim _{k \rightarrow \infty} \frac{1}{d^{k}} \sum_{\substack{\varphi^{k}([w: 1])=\alpha \\ F(w) \neq 0}} \log |F(w)|_{v} .
$$

where the $[w: 1]$ for which $\varphi^{k}([w: 1])=\alpha$ are counted with multiplicity. 
Proof. The polynomial $F$ factors as $F(t)=\gamma \prod_{i=1}^{n}\left(t-\beta_{i}\right)$ where $\gamma$ and $\beta_{1}, \ldots, \beta_{n}$ are elements of $\bar{K}$. For each $\beta_{i}$, the multiplicity of $\beta_{i}$ in $\left(\varphi^{k}\right)^{*} \alpha$ is at most $r\left(\left(\varphi^{k}\right)^{*} \alpha\right)$ (where $r\left(\left(\varphi^{k}\right)^{*} \alpha\right.$ ) is defined as in Section 3). Since $\alpha$ is not exceptional, we have $\lim _{k \rightarrow \infty} \frac{r\left(\left(\varphi^{k}\right)^{*} \alpha\right)}{d^{k}}=0$, by Lemma 4.1. Thus,

$$
\lim _{k \rightarrow \infty} \frac{1}{d^{k}} \sum_{\substack{\varphi^{k}([w: 1])=\alpha \\
w \neq \beta_{j}}} \log \left|w-\beta_{j}\right|_{v}=\lim _{k \rightarrow \infty} \frac{1}{d^{k}} \sum_{\begin{array}{r}
\varphi^{k}([w: 1])=\alpha \\
F(w) \neq 0
\end{array}} \log \left|w-\beta_{j}\right|_{v}
$$

for each $\beta_{j}$. Hence, it suffices to show that

$$
\int_{\mathbb{P}^{1}\left(\mathbb{C}_{v}\right)} \log |t-\beta|_{v} d \mu_{\varphi, v}=\lim _{k \rightarrow \infty} \frac{1}{d^{k}} \sum_{\substack{\varphi^{k}([w: 1])=\alpha \\ w \neq \beta}} \log |w-\beta|_{v}
$$

for any $\beta \in \bar{K}$.

Note that $\varphi^{k}([w: 1])=[s: u]$ if and only if $u P_{k}(w, 1)-s Q_{k}(w, 1)=0$. Thus, as polynomials in $t$, we have

$$
u P_{k}(t, 1)-s Q_{k}(t, 1)=\eta_{k} \prod_{\varphi^{k}([w: 1])=[s: u]}(t-w),
$$

where $\eta_{k} \in \bar{K}$. We write

$$
u P_{k}(t, 1)-s Q_{k}(t, 1)=(t-\beta)^{w_{k}} G_{k}(t, 1)
$$

for a polynomial $G_{k}$ such that $G_{k}(\beta, 1) \neq 0$, as in Proposition 4.4. Note that

$$
G_{k}(t, 1)=\eta_{k} \prod_{\substack{\varphi^{k}([w: 1])=\alpha \\ w \neq \beta}}(t-w)
$$

Plugging $\beta$ in for $t$ and taking logs of absolute values gives

$$
\log \left|G_{k}(\beta, 1)\right|_{v}=\log \left|\eta_{k}\right|_{v}+\sum_{\substack{\varphi^{k}([w: 1])=[s: u] \\ w \neq \beta}} \log |w-\beta|_{v} .
$$

Applying Proposition 4.4 therefore yields

$$
\begin{aligned}
\lim _{k \rightarrow \infty} \frac{1}{d^{k}} & \sum_{\substack{\varphi^{k}([w: 1])=\alpha \\
w \neq \beta}} \log |w-\beta|_{v}+\frac{\log \left|\eta_{k}\right|_{v}}{d^{k}} \\
& =\lim _{k \rightarrow \infty} \frac{\log \max \left(\left|P_{k}(\beta, 1)\right|_{v},\left|Q_{k}(\beta, 1)\right|_{v}\right)}{d^{k}} .
\end{aligned}
$$

Now, writing

$$
u P_{k}\left(T_{0}, T_{1}\right)-s Q_{k}\left(T_{0}, T_{1}\right)=T_{1}^{w_{k}} V_{k}\left(T_{0}, T_{1}\right)
$$

for some polynomial $V_{k}$ such that $V_{k}(1,0) \neq 0$, we see that $\eta_{k}=V_{k}(1,0)$. Applying Proposition 4.4, we obtain

$$
\lim _{k \rightarrow \infty} \frac{\log \left|\eta_{k}\right|_{v}}{d^{k}}=\lim _{k \rightarrow \infty} \frac{\log \max \left(\left|P_{k}(1,0)\right|_{v},\left|Q_{k}(1,0)\right|_{v}\right)}{d^{k}} .
$$


Substituting this equality into (4.6.3) gives

$$
\begin{aligned}
\lim _{k \rightarrow \infty} \frac{1}{d^{k}} \sum_{\substack{\varphi^{k}([w: 1])=\alpha \\
[w: 1] \neq \beta}} \log |w-\beta|_{v} & =\lim _{k \rightarrow \infty} \frac{\log \max \left(\left|P_{k}(\beta, 1)\right|_{v},\left|Q_{k}(\beta, 1)\right|_{v}\right)}{d^{k}} \\
& -\lim _{k \rightarrow \infty} \frac{\log \max \left(\left|P_{k}(1,0)\right|_{v},\left|Q_{k}(1,0)\right|_{v}\right)}{d^{k}} .
\end{aligned}
$$

Using Proposition 2.1, we obtain (4.6.1).

Now, we show that the same result holds when we average $\log |\beta-w|_{v}$ over periodic points rather than inverse images of a point.

Theorem 4.7. For any any polynomial $F \in \bar{K}[t]$ we have

$$
\int_{\mathbb{P}^{1}\left(\mathbb{C}_{v}\right)} \log |F|_{v} d \mu_{\varphi, v}=\lim _{k \rightarrow \infty} \frac{1}{d^{k}} \sum_{\substack{\varphi^{k}([w: 1])=[w: 1] \\ F(w) \neq 0}} \log |F(w)|_{v},
$$

where the $[w: 1]$ for which $\varphi^{k}([w: 1])=w$ are counted with multiplicity.

Proof. As in the proof of Theorem 4.6, it will suffice to show that

$$
\int_{\mathbb{P}^{1}\left(\mathbb{C}_{v}\right)} \log |t-\beta|_{v} d \mu_{\varphi, v}=\lim _{k \rightarrow \infty} \frac{1}{d^{k}} \sum_{\substack{\varphi^{k}([w: 1])=[w: 1] \\ w \neq \beta}} \log |w-\beta|_{v}
$$

for any $\beta \in \bar{K}$ (this follows from the fact that the multiplicity of each $\beta_{i}$ as a $k$-periodic point is bounded for all $k$ by Proposition 4.5).

We have $\varphi^{k}([w: 1])=[w: 1]$ if and only if $P_{k}(w, 1)-w Q_{k}(w, 1)=0$. Thus,

$$
P_{k}(t, 1)-t Q_{k}(t, 1)=\gamma_{k} \prod_{\varphi^{k}([w: 1])=[w: 1]}(t-w),
$$

for some $\gamma_{k} \in \bar{K}$. We write

$$
P_{k}(t, 1)-t Q_{k}(t, 1)=(t-\beta)^{n_{k}} H_{k}(t, 1)
$$

for a polynomial $H_{k}$ such that $H_{k}(\beta, 1) \neq 0$. We have

$$
H_{k}(t, 1)=\gamma_{k} \prod_{\substack{\varphi^{k}([w: 1])=[w: 1] \\ w \neq \beta}}(t-w) .
$$

Then, plugging $\beta$ in for $t$, taking logs of absolute values, and applying Proposition 4.5 gives

$$
\begin{aligned}
\lim _{k \rightarrow \infty} \frac{1}{d^{k}} \sum_{\substack{\varphi^{k}([w: 1])=[w: 1] \\
w \neq \beta}} \log |\beta-w|_{v}+\frac{\log \left|\gamma_{k}\right|_{v}}{d^{k}} \\
\quad=\lim _{k \rightarrow \infty} \frac{\log \max \left(\left|P_{k}(\beta, 1)\right|_{v},\left|Q_{k}(\beta, 1)\right|_{v}\right)}{d^{k}}
\end{aligned}
$$


Writing

$$
T_{1} P_{k}\left(T_{0}, T_{1}\right)-T_{0} Q_{k}\left(T_{0}, T_{1}\right)=T_{1}^{n_{k}} W_{k}\left(T_{0}, T_{1}\right)
$$

for a polynomial $W_{k}$ such that $W_{k}(1,0) \neq 0$, we see that $\gamma_{k}=W_{k}(1,0)$. By Proposition 4.5, we have

$$
\lim _{k \rightarrow \infty} \frac{\log \left|\gamma_{k}\right|_{v}}{d^{k}}=\lim _{k \rightarrow \infty} \frac{\log \max \left(\left|P_{k}(1,0)\right|_{v},\left|Q_{k}(1,0)\right|_{v}\right)}{d^{k}} .
$$

Combining this equality with (4.7.2) and Proposition 2.1 yields (4.7.1).

We are now ready to prove the results regarding the computation of the canonical height $h_{\varphi}(\beta)$. First, we'll need a lemma. Note that the lemma does not follow directly from the work of Call an Goldstine ([CG97]), since they only prove that in a fixed number field, the local canonical heights sum to the global canonical height. What is required here is slightly different.

Lemma 4.8. Let $\beta=[a: b]$ in $\mathbb{P}^{1}(\bar{K})$. Let $\left[a_{1}: b_{1}\right], \ldots,\left[a_{n}: b_{n}\right]$ be the conjugates of $[a: b]$ under the action of $\operatorname{Gal}(\bar{K} / K)$. Then

$$
\begin{aligned}
{[K(\beta): K] } & (\operatorname{deg} K) h_{\varphi}([a: b]) \\
& =\sum_{\text {places v of } K} \lim _{k \rightarrow \infty} \sum_{i=1}^{n} \frac{\log \max \left(\left|P_{k}\left(a_{i}, b_{i}\right)\right|_{v},\left|Q_{k}\left(a_{i}, b_{i}\right)\right|_{v}\right)}{d^{k}} .
\end{aligned}
$$

Proof. For all but finitely many $v$, we have $\left|a_{i}\right|_{v}=\left|b_{i}\right|_{v}=1$. Furthermore, for all but finitely many $v$, we have

$$
\log \max \left(\left|P_{k}(s, t)\right|_{v},\left|Q_{k}(s, t)\right|_{v}\right)=0
$$

for all $k$ whenever $|s|_{v}=|t|_{v}=1$. This is true, for example, at all nonarchimedean $v$ of good reduction for $\varphi$ in the sense of [PST04]. Indeed, when $v$ is a finite place, (4.8.2) will hold for all $|s|_{v}=|t|_{v}=1$ unless either $\left|\operatorname{Res}\left(P\left(T_{0}, 1\right), Q\left(T_{0}, 1\right)\right)\right|_{v}$ or $\left|\operatorname{Res}\left(P\left(1, T_{1}\right), Q\left(1, T_{1}\right)\right)\right|_{v}$ is less than 1 , where Res is the usual resultant of two polynomials (see [BK86, p. 279, Proposition 4]). Thus, we can interchange the limit and the sum on the right-hand side of (4.8.1) so that

$$
\begin{aligned}
\lim _{k \rightarrow \infty} & \sum_{\text {places } v \text { of }} \sum_{i=1}^{n} \frac{\log \max \left(\left|P_{k}\left(a_{i}, b_{i}\right)\right|_{v},\left|Q_{k}\left(a_{i}, b_{i}\right)\right|_{v}\right)}{d^{k}} \\
= & \sum_{\text {places } v \text { of } K} \lim _{k \rightarrow \infty} \sum_{i=1}^{n} \frac{\log \max \left(\left|P_{k}\left(a_{i}, b_{i}\right)\right|_{v},\left|Q_{k}\left(a_{i}, b_{i}\right)\right|_{v}\right)}{d^{k}} .
\end{aligned}
$$

Now, let $L$ be the field $K(\beta)$ and let $w$ be a place of $L$ that extends the place $v$ of $K$; we write $w \mid v$. The field $L$ has $n$ embeddings $i: L \hookrightarrow \mathbb{C}_{v}$; for exactly $\left[L_{w}: K_{v}\right]$ of these embeddings, we have $|i(x)|_{v}=|x|_{w}$ for all $x \in L$. 
This yields $\left[L_{w}: K_{v}\right]$ conjugates $\left[a^{\prime}: b^{\prime}\right]$ of $[a: b]$ such that $|a|_{w}=\left|a^{\prime}\right|_{v}$ and $|b|_{w}=\left|b^{\prime}\right|_{w}$. Hence, we see that

$$
\begin{aligned}
& \sum_{i=1}^{n} \log \max \left(\left|P_{k}\left(a_{i}, b_{i}\right)\right|_{v},\left|Q_{k}\left(a_{i}, b_{i}\right)\right|_{v}\right) \\
& \quad=\sum_{w \mid v}\left[L_{w}: K_{v}\right] \log \max \left(\left|P_{k}(a, b)\right|_{w},\left|Q_{k}(a, b)\right|_{v}\right) .
\end{aligned}
$$

Thus, we have

$$
\begin{array}{r}
\sum_{\text {places } v \text { of } K} \sum_{i=1}^{n} \log \max \left(\left|P_{k}\left(a_{i}, b_{i}\right)\right|_{v},\left|Q_{k}\left(a_{i}, b_{i}\right)\right|_{v}\right) \\
=[K(\beta): K](\operatorname{deg} K) h\left(\varphi^{k}([a: b])\right),
\end{array}
$$

by (1.0.6). It follows from (1.0.7) and (4.8.3) that we therefore have

$$
\begin{aligned}
\sum_{\text {places } v \text { of } K} \lim _{k \rightarrow \infty} \sum_{i=1}^{n} \frac{\log \max \left(\left|P_{k}\left(a_{i}, b_{i}\right)\right|_{v},\left|Q_{k}\left(a_{i}, b_{i}\right)\right|_{v}\right)}{d^{k}} \\
=[K(\beta): K](\operatorname{deg} K) \lim _{k \rightarrow \infty} \frac{h\left(\varphi^{k}([a: b])\right)}{d^{k}} \\
=[K(\beta): K](\operatorname{deg} K) h_{\varphi}([a: b]) .
\end{aligned}
$$

Theorem 4.9. Let $\alpha$ be any point in $\mathbb{P}^{1}(\bar{K})$ that is not an exceptional point of $\varphi$. Then, for any $\beta \in \bar{K}$ and any nonzero irreducible $F \in K[t]$ such that $F(\beta)=0$, we have

$$
\begin{aligned}
& (\operatorname{deg} K)(\operatorname{deg} F)\left(h_{\varphi}(\beta)-h_{\varphi}(\infty)\right) \\
& =\sum_{\text {places } v \text { of } K} \lim _{k \rightarrow \infty} \frac{1}{d^{k}} \sum_{\begin{array}{c}
k \\
\varphi^{k}([w: 1])=\alpha \\
F(w) \neq 0
\end{array}} \log |F(w)|_{v},
\end{aligned}
$$

where the $[w: 1]$ for which $\varphi^{k}([w: 1])=\alpha$ are counted with multiplicity.

Proof. Write $F(t)=\gamma \prod_{i=1}^{n}\left(t-\beta_{i}\right)$ where $\gamma \in K$ and the $\beta_{i}$ are the conjugates of $\beta$ under the action of $\operatorname{Gal}(\bar{K} / K)$. By the product formula, we have $\sum_{\text {places } v \text { of } K} \log |\gamma|_{v}=0$. Thus, using Theorem 4.6 and Proposition 2.1, we 
see that

$$
\begin{aligned}
& \sum_{\text {places } v \text { of } K} \lim _{\substack{k \rightarrow \infty \\
d^{k}}} \frac{1}{\substack{\varphi^{k}([w: 1])=\alpha \\
F(w) \neq 0}} \log |F(w)|_{v} \\
= & \sum_{\text {places } v \text { of } K} \lim _{\substack{k \rightarrow \infty \\
d^{k}}} \frac{1}{\substack{\varphi^{k}([w: 1])=\alpha \\
F(w) \neq 0}} \log \left|\prod_{i=1}^{n}\left(w-\beta_{i}\right)\right|_{v} \\
= & \sum_{i=1}^{n} \lim _{k \rightarrow \infty} \frac{\log \max \left(\left|P_{k}\left(\beta_{i}, 1\right)\right|_{v},\left|Q_{k}\left(\beta_{i}, 1\right)\right|_{v}\right)}{d^{k}} \\
- & (\operatorname{deg} F) \lim _{k \rightarrow \infty} \frac{\log \max \left(\left|P_{k}(1,0)\right|_{v},\left|Q_{k}(1,0)\right|_{v}\right)}{d^{k}}
\end{aligned}
$$

By Lemma 4.8, the quantity on the last two lines is equal to

$$
(\operatorname{deg} F)(\operatorname{deg} K)\left(h_{\varphi}(\beta)-h_{\varphi}(\infty)\right)
$$

as desired.

Theorem 4.10. For any $\beta \in \bar{K}$ and any nonzero irreducible $F \in K[t]$ such that $F(\beta)=0$, we have

$$
\begin{aligned}
& (\operatorname{deg} K)(\operatorname{deg} F)\left(h_{\varphi}(\beta)-h_{\varphi}(\infty)\right) \\
& =\sum_{\text {places } v \text { of } K} \lim _{k \rightarrow \infty} \frac{1}{d^{k}} \sum_{\substack{\varphi^{k}([w: 1])=[w: 1] \\
F(w) \neq 0}} \log |F(w)|_{v},
\end{aligned}
$$

where the $[w: 1]$ for which $\varphi^{k}([w: 1])=w$ are counted with multiplicity.

Proof. The proof is the same as the proof of Theorem 4.9, using Theorem 4.7 in place of Theorem 4.6.

\section{A counterexample}

The main theorems of this paper are not true when we work over the complex numbers $\mathbb{C}$ rather than $\bar{K}$. Let $K=\mathbb{Q}$ and let $\varphi([x: y])=\left[x^{2}\right.$ : $\left.y^{2}\right]$ be the usual squaring map. Let $v$ be the archimedean place of $\mathbb{Q}$, so that $\mathbb{C}_{v}$ is just the usual complex numbers $\mathbb{C}$. We define the function $\psi$ on the positive integers recursively by $\psi(1)=2$ and $\psi(n)=2^{(n \psi(n-1))}$. Let $\alpha=\sum_{n=1}^{\infty} 1 / \psi(n)$ and let $\beta=e^{2 \pi i \alpha}$. Note that for any $t$, we have $\left|e^{2 \pi i t}-1\right| \leq \pi(t-[t])$, (where $[t]$ is the greatest integer less than or equal to 
$t$ ). Letting $\ell_{n}=\log _{2} \psi(n)$, we then have

$$
\begin{aligned}
& \frac{1}{2^{\ell_{n}}} \sum_{w^{2^{\ell_{n}}}=1} \log |w-\beta|_{v}=\frac{\log \left|\beta^{\psi(n)}-1\right|}{\psi(n)} \\
& \leq \frac{1}{\psi(n)} \log (\pi(\psi(n) \alpha-[\psi(n) \alpha])) \\
& \leq \frac{1}{\psi(n)} \log \left(\pi \frac{\psi(n)}{\psi(n+1)} \sum_{j=0}^{\infty} \frac{1}{2^{j \psi(n+1)}}\right) \\
& \leq \log \pi+1-n \log 2+\log 2 .
\end{aligned}
$$

Thus, $\frac{1}{2^{\ell_{n}}} \sum_{w^{2} \ell_{n}}=1 \log |\beta-w|_{v}$ goes to $-\infty$ as $n \rightarrow \infty$, so

$$
\lim _{k \rightarrow \infty} \frac{1}{2^{k}} \sum_{w^{2^{k}}=1} \log |w-\beta|_{v}
$$

does not exist.

\section{Applichtions AND FURTher QUestions}

6.1. Lyapunov exponents. The Lyapunov exponent $L(\varphi)$ of a rational $\operatorname{map} \varphi: \mathbb{P}_{\mathbb{C}}^{1} \longrightarrow \mathbb{P}_{\mathbb{C}}^{1}$ (see [Mañ88]) can be defined as follows. Choosing coordinates $\left[T_{0}: T_{1}\right]$ for $\mathbb{P}_{\mathbb{C}}^{1}$, letting $t=T_{0} / T$, and writing $\varphi(t)=P(t) / Q(t)$ for polynomials $P$ and $Q$, we define

$$
L(\varphi)=\int_{\mathbb{P}^{1}(\mathbb{C})} \log \left|\varphi^{\prime}(t)\right| d \mu_{\varphi},
$$

where $\mu_{\varphi}$ is the unique measure of maximal entropy measure for $\varphi$ on $\mathbb{P}^{1}$; this measure of maximal entropy is the same as the Brolin-Lyubich measure discussed in Section 2 (see [Mañ83]).

The Lyapunov exponent can be computed via equidistribution on certain subsequences of inverse images of nonexceptional points in $\mathbb{P}^{1}(\mathbb{C})$ (see [DeM03], [Mañ88]). That is, given a nonexceptional point $\alpha$ in $\mathbb{P}^{1}(\mathbb{C})$, there is an infinite strictly increasing sequence of integers $\left(m_{i}\right)_{i=1}^{\infty}$ such that

$$
L(\varphi)=\lim _{i \rightarrow \infty} \frac{1}{(\operatorname{deg} \varphi)^{m_{i}}} \sum_{\substack{\varphi^{m_{i}(\beta)=\alpha} \\ \varphi^{\prime}(\beta) \neq 0 \\ \beta \neq \infty}} \log \left|\varphi^{\prime}(\beta)\right| .
$$

It is not known, however, if $L(\varphi)$ can be computed by taking the limit of the average $\varphi^{\prime}$ on the periodic points of $\varphi$.

When $\varphi$ is defined over a number field $K$, however, we obtain the following result as a corollary of Theorem 4.7.1.

Corollary 6.1. Let $K$ be a number field and let $\varphi: \mathbb{P}_{\mathbb{C}}^{1} \longrightarrow \mathbb{P}_{\mathbb{C}}^{1}$ be a nonconstant rational map that is defined via base extension from a map 
$\varphi: \mathbb{P}_{K}^{1} \longrightarrow \mathbb{P}_{K}^{1}$. Let $\varphi^{\prime}$ be defined as above. Then

$$
L(\varphi)=\lim _{k \rightarrow \infty} \frac{1}{(\operatorname{deg} \varphi)^{k}} \sum_{\substack{\varphi^{k}(\xi)=\xi \\ \varphi^{\prime}(\xi) \neq 0 \\ \xi \neq \infty}} \log \left|\varphi^{\prime}(\xi)\right| .
$$

Proof. We may write $\varphi^{\prime}$ as a quotient of polynomials $A(t) / B(t)$ with coefficients in $K$. This yields $\log \left|\varphi^{\prime}(t)\right|=\log |A(t)|-\log |B(t)|$. The corollary then follows immediately from Theorem 4.7.

This corollary says that if $\varphi$ is a rational function defined over a number field, then the Lyapunov exponent of $\varphi$ is completely determined by the derivative of $\varphi$ at the periodic points of $\varphi$. This means that the derivative of $\varphi$ at the periodic points of $\varphi$ also determines the Hausdorff dimension of the Julia set (see [FLM83]).

6.2. Symmetry of canonical heights. In [ST], we show that when $\infty$ is not in the $v$-adic Julia set of $\varphi$ for any archimedean $v$, we have

$$
\lim _{k \rightarrow \infty} \frac{1}{d^{k}} \sum_{\varphi^{k}([w: 1])=[w: 1]} h(w)=\lim _{\ell \rightarrow \infty} \frac{1}{2^{\ell}} \sum_{\xi^{2^{\ell}=\xi}} h_{\varphi}(\xi) .
$$

This can be thought of as a symmetry relation, connecting $h$ of the $\varphi$ periodic points with $h_{\varphi}$ of the roots of unity. The proof uses Theorem 4.10 along with Lyubich's equidistribution theorem ([Lyu83]) and some adelic intersection theory (see [Zha95] and [Zha92]). We are also able to use Theorem 4.10 to prove that

$$
h_{\varphi}(\beta)-h(\beta) \leq \lim _{k \rightarrow \infty} \frac{1}{d^{k}} \sum_{\varphi^{k}([w: 1])=[w: 1]} h(w)+h_{\varphi}(\infty)+\log 2 .
$$

Our proof of (6.1.1) does not work when $\infty$ is in the $v$-adic Julia set of $\varphi$, for in that case the local height $\hat{h}_{v}$ is not bounded on the $v$-adic Julia set. Unfortunately, the $v$-adic Julia set is all of $\mathbb{P}^{1}\left(\mathbb{C}_{v}\right)$ when $v$ is archimedean for many rational maps $\varphi$. This is the case, for example, when $\varphi$ is the map obtained by taking the multiplication-by- 2 map on an elliptic curve and modding out by the hyperelliptic involution (such a map is called a Lattès map).

On the other hand, the usual local height $\hat{h}_{v}(t)$ of an element $t \in \mathbb{C}_{v}$ is simply $\max \left(\log |t|_{v}, 0\right)$, which is only a little bit different from $\log |t|_{v}$, and Theorem 4.7 proves a suitable equidistribution theorem for $\log |t|_{v}$. We hope to extend the techniques of this paper so that we can prove an analog of Theorem 4.7 for functions such as $\max \left(\log |t|_{v}, 0\right)$.

6.3. Computing with points of small height. The results in [Bil97], [Aut01], [BR06], [FRL04], [FRL07], and [CL06] all apply not only to the periodic points and backwards iterates of a point that we treat in this paper but to all points of small height in the algebraic closure of a number field 
$K$. For example, one the main theorems in [BR06], [FRL04], [FRL07], and [CL06] states that for any continuous function $g$ on $\mathbb{P}^{1}\left(\mathbb{C}_{v}\right)$ and any infinite nonrepeating sequence of points $\left(\alpha_{n}\right)$ in $\mathbb{P}^{1}(\bar{K})$ such that $\lim _{n \rightarrow \infty} h_{\varphi}\left(\alpha_{n}\right)=$ 0 , one has

$$
\lim _{n \rightarrow \infty} \frac{1}{\left|\operatorname{Gal}\left(\alpha_{n}\right)\right|} \sum_{\sigma \in \operatorname{Gal}\left(\alpha_{n}\right)} g\left(\alpha_{n}^{\sigma}\right)=\int_{\mathbb{P}^{1}\left(\mathbb{C}_{v}\right)} g d \mu_{v, \varphi},
$$

where $\operatorname{Gal}\left(\alpha_{n}\right)$ is the Galois group of the Galois closure of $K\left(\alpha_{n}\right)$ over $K$.

Baker, Ih, and Rumely ([BIR05]) and Autissier ([Aut07]) have produced counterexamples that show that (6.1.2) does not always hold when the function $g$ is replaced with $\log |F|_{v}$ for $F$ a polynomial. All of these examples involve infinite nonrepeating sequences of points $\left(\alpha_{n}\right) \in \overline{\mathbb{Q}}$ such that $\lim _{n \rightarrow \infty} h\left(\alpha_{n}\right)=0$ and

$$
\lim _{n \rightarrow \infty} \frac{1}{\left|\operatorname{Gal}\left(\alpha_{n}\right)\right|} \sum_{\sigma \in \operatorname{Gal}\left(\alpha_{n}\right)} \log \left|\alpha_{n}^{\sigma}-2\right| \neq \int_{0}^{1} \log \left|e^{2 \pi i \theta}-2\right| d \theta .
$$

The points $\left(\alpha_{n}\right)$ are not preperiodic in any of these examples Thus, it may be possible to prove that the main results of this paper continue to hold when we work with any nonrepeating sequence of Galois orbits of preperiodic points. This would imply the following conjectured generalization of Siegel's theorem for integral points.

Conjecture 6.2 (Ih). For any nonpreperiodic point $\beta \in \mathbb{P}_{\mathfrak{o}_{K}}^{1}(\bar{K})$, there are at most finitely many preperiodic points of $\varphi$ in $\mathbb{P}_{\mathfrak{o}_{K}}^{1}(\bar{K})$ that are integral relative to $\beta$. (Here, $\mathfrak{o}_{K}$ is the ring of integers of $K$ and $\alpha$ is said to be integral relative to $\beta$ if the Zariski closure of $\alpha$ does not meet the Zariski closure of $\beta$ in $\mathbb{P}_{\mathfrak{o}_{K}}^{1}$.)

Baker, Ih, and Rumely have proven that this is true when $\varphi$ is a Lattès map or the usual squaring map $x \mapsto x^{2}$. Using Theorem 4.10 and arguing as in [BIR05] (or as in [Sil93], which presents a related result), it is possible to derive the following weak version of Ih's conjecture in general.

Proposition 6.3. For any nonpreperiodic point $\beta \in \mathbb{P}^{1}(\bar{K})$, there are at most finitely many $n$ such that all $\alpha \in \mathbb{P}^{1}(\bar{K})$ of period $n$ are $\beta$-integral.

\section{REFERENCES}

[Aut01] P. Autissier, Points entiers sur les surfaces arithmétiques, J. reine. angew. Math 531 (2001), 201-235.

[Aut07] _ Sur une question d'equir'epartition de nombres algébriques, C. R. Acad. Sci. Paris (2007), to appear.

[Bak75] A. Baker, Transcendental number theory, Cambridge University Press, Cambridge, 1975.

[Bea91] A. F. Beardon, Iteration of rational functions, Springer-Verlag, New York, 1991.

[Ber90] V. G. Berkovich, Spectral theory and analytic geometry over nonarchimedean fields, AMS Mathematical Surveys and Monographs, American Mathematical Society, Providence, 1990. 
[Bil97] Y. Bilu, Limit distribution of small points on algebraic tori, Duke Math 89 (1997), 465-476.

[BIR05] M. Baker, S. I. Ih, and R. Rumely, A finiteness property of torsion points, 2005, preprint, 30 pages.

[BK86] E. Brieskorn and H. Knörrer, Plane algebraic curves, Birkhäuser, Basel, 1986, Translated by J. Stillwell.

[BR04] M. Baker and R. Rumely, Analysis and dynamics on the Berkovich projective line, preprint. Available at arxiv:math.NT/0407433, 150 pages., 2004.

[BR06] _ Equidistribution of small points, rational dynamics, and potential theory, Ann. Inst. Fourier (Grenoble) 56 (2006), 625-688.

[Bro65] H. Brolin, Invariant sets under iteration of rational functions, Ark. Mat. 6 (1965), 103-144.

[CG97] G. S. Call and S. Goldstine, Canonical heights on projective space, J. Number Theory 63 (1997), 211-243.

[CL06] A. Chambert-Loir, Mesures et équidistribution sur les espaces de Berkovich, preprint. Available at arxiv:abs/math.NT/0304023, 20 pages, 2006.

[CLT04] A. Chambert-Loir and A. Thuillier, Formule de mahler et équidistribution logarithmique, preprint. Available at arxiv:abs/math.NT/0612556, 24 pages, 2004.

[CS93] G. S. Call and J. Silverman, Canonical heights on varieties with morphism, Compositio Math. 89 (1993), 163-205.

[Dav95] S. David, Minorations de formes linéaire de logarithmes elliptiques, Mem. Soc. Math. France 62 (1995), 143 pp.

[DeM03] L. DeMarco, Dynamics of rational maps: Lyapunov exponents, bifurcations, and capacity, Math. Ann. 203 (2003), 43-73.

[EF96] G. Everest and Bríd Ní Fhlathúin, The elliptic mahler measure, Math. Proc. Cambridge Philos. Soc. 120 (1996), 13-25.

[EW99] G. Everest and T. Ward, Heights of Polynomials and Entropy in Algebraic Dynamics, Springer-Verlag, New York, 1999.

[FLM83] A. Freire, A. Lopes, and R. Mañe, An invariant measure for rational functions, Boletim da Sociedade Brasileira de Matematica 14 (1983), 45-62.

[FRL04] C. Favre and J. Rivera-Letelier, Théorème d'équidistribution de Brolin en dynamique p-adique, C. R. Math. Acad. Sci. Paris 339 (2004), no. 4, 271-276.

[FRL07] _ Équidistribution des points de petite hauteur, Math. Ann. (2007), to appear, 46 pages.

[Lan83] S. Lang, Fundamentals of diophantine geometry, Springer-Verlag, New York, 1983.

[Lyu83] M. Lyubich, Entropy properties of rational endomorphisms of the Riemann sphere, Ergodic Theory Dynam. Systems 3 (1983), 351-385.

[Mah60] K. Mahler, An application of Jensen's formula to polynomials, Mathematica 7 (1960), 98-100.

[Mañ83] Ricardo Mañé, On the uniqueness of the maximizing measure for rational maps, Bol. Soc. Brasil. Mat. 14 (1983), no. 1, 27-43.

[Mañ88] R. Mañe, The Hausdorff dimension of invariant probabilities of rational maps, Dynamical Systems, Valparaiso 1986 (R. Bamon, R. Labarca, and J. Palis, eds.), Springer-Verlag, 1988, pp. 86-117.

[Mil99] J. Milnor, Dynamics in one complex variable, Vieweg, Braunschweig, 1999.

[MS95] P. Morton and J. H. Silverman, Periodic points, multiplicities, and dynamical units, J. Reine Angew. Math. 461 (1995), 81-122.

[Piñ05] J. Piñeiro, Mahler formula for morphisms on $\mathbb{P}^{n}$, Ph.D. thesis, City University of New York, 2005.

[PST04] J. Piñeiro, L. Szpiro, and T. Tucker, Mahler measure for dynamical systems on $\mathbb{P}^{1}$ and intersection theory on a singular arithmetic surface, Geometric methods 
in algebra and number theory (F. Bogomolov and Y. Tschinkel, eds.), Progress in Mathematics 235, Birkhäuser, 2004, pp. 219-250.

[Rot55] K. F. Roth, Rational approximations to algebraic numbers, Mathematika 2 (1955), 1-20, corrigendum, ibid. 2 (1955), 168.

[Sch74] A. Schinzel, Primitive divisors of the expression $a^{n}-b^{n}$ in algebraic number fields, J. Reine Angew. 268/269 (1974), 27-33, Collection of articles dedicated to Helmut Hasse on his seventy-fifth birthday, II.

[Sie29] C. L. Siegel, Über einige Anwendungen diophantische Approximationen, Abh. Preuss. Akad. Wiss. Phys. Math. Kl. (1929), 41-69.

[Sil93] J. H. Silverman, Integer points, Diophantine approximation, and iteration of rational maps, Duke Math. J. 71 (1993), no. 3, 793-829.

[ST] L. Szpiro and T. Tucker, Symmetry of canonical heights of periodic points, In preparation.

[SUZ97] L. Szpiro, E. Ullmo, and S. Zhang, Equirépartition des petits points, Invent. Math. 127 (1997), 337-347.

[Thu06] A. Thuillier, Théorie de potential sur les courbes en géométrie non archimédienne, applications à la théorie d'arakelov, Ph.D. thesis, Université ded Rennes 1, 2006.

[Zha92] S. Zhang, Positive line bundles on arithmetic surfaces, Annals of Math 136 (1992), 569-587.

[Zha95] Small points and adelic metrics, J. Algebraic Geometry 4 (1995), 281300.

Lucien Szpiro, Ph.D. Program in Mathematics, Graduate Center of CUNy, 365 Fifth Avenue, New York, NY 10016-4309

E-mail address: lszpiro@gc.cuny.edu

Thomas Tucker, Department of Mathematics, Hylan Building, University of Rochester, Rochester, NY 14627

E-mail address: ttucker@math.rochester.edu 